Henry et al., 09-17-2020 bioRxiv

\title{
GxGxE interactions and adaptive potential of the microbiome in Drosophila melanogaster
}

\author{
Lucas P. Henry ${ }^{1,2}$, Michael Fernandez ${ }^{1,2}$, Julien F. Ayroles ${ }^{1,2}$ \\ ${ }^{1}$ Dept. of Ecology and Evolutionary Biology, Princeton University \\ ${ }^{2}$ Lewis Sigler Institute for Integrative Genomics, Princeton University \\ email:LPH Ihenry@princeton.edu ; JFA jayroles@princeton.edu
}

\begin{abstract}
The microbiome may serve as a reservoir of adaptive potential if hosts can leverage microbial adaptations in stressful environments. However, the facilitation of rapid host adaptation may be limited by interactions between host genotype, microbiome, and environment $\left(\mathrm{G}_{\mathrm{H}} \times \mathrm{G}_{\mathrm{M}} \times \mathrm{E}\right)$. Here, to understand how host $\mathrm{x}$ microbiome $\mathrm{x}$ environment interactions shape adaptation, we leverage $>150$ generations of experimental evolution in Drosophila melanogaster in a stressful environment, high sugar (HS) diet. The microbiome of HS adapted flies shifted to be dominated by one bacteria, Acetobacter pasteurianus. We next performed a fully reciprocal transplant experiment using the dominant control and HS bacteria to measure life-history and metabolic traits in flies. Mismatches between fly evolution and microbiome exerted fitness costs by slowing development and reducing fecundity, especially in the stressful HS diet. The $\mathrm{G}_{H} \times \mathrm{G}_{\mathrm{M}} \times \mathrm{E}$ interactions observed suggest that both ecological context and host evolution shape the adaptive potential of the microbiome. We conclude by proposing future directions that highlight the benefit of using experimental evolution to study the contribution of the microbiome to host evolution.
\end{abstract}

\section{INTRODUCTION}

The microbiome has profound effects on many aspects of organismal biology. From modulating physiology [1] to pathogen protection [2] to social interactions [3], the microbiome is frequently implicated as a key regulator of many host phenotypes--but can the microbiome also influence host adaptation? For hosts where microbes are tightly controlled and faithfully transmitted across generations, microbes have clear influence on host evolution $[4,5]$. For example, the acquisition of nutrition-provisioning symbionts enables the ecological expansion of sap-feeding insects [6]. For many eukaryotes, though, their microbiomes are environmentally acquired or through other weakly controlled transmission mechanisms [5]. Weak control over microbial transmission disrupts the links between the microbiome and host over generations. Without these links, the microbiome may have limited effects on host evolution $[4,7]$.

Microbiomes may still have the potential to influence host evolution because environmentally acquired microbes must survive and adapt to external environments. In doing so, microbiomes may acquire beneficial traits that hosts can leverage. Thus, microbiomes have the potential to precede and facilitate rapid host adaptation, but this process is poorly understood.

The environmentally acquired microbiome appears to facilitate rapid host adaptation in several systems. For example, bean beetles acquire pesticide resistance from their soil-dwelling Burkholderia symbiont [8]. The gut microbiome of woodrats facilitates survival on toxic plant diets [9]. In plants, drought-adapted soil communities can increase fitness in drought conditions [10]. These studies suggest that microbes can adapt and evolve to stressful conditions in natural environments and buffer the stressor for the host, but much about the evolution of these interactions remains unclear--specifically, we do not know to what extent these microbial effects depend on host genotypes. Host genetic variation may influence the responsiveness to microbial variation such that some combinations of host and microbiome differentially affect host phenotypes within species. The effects on host phenotype will also likely depend on the environmental context, where mismatches between the environment, host genotype, and microbiome may negatively affect host fitness. In other words, host genotype x microbial genotype $\mathrm{x}$ environment ( $\mathrm{GH}$ x GM $\mathrm{x}$ E) interactions may shape how host-microbiome systems evolve. Such complex $\mathrm{G}_{\mathrm{H}} \mathrm{x} \mathrm{G}_{\mathrm{M}}$ $\mathrm{x}$ E interactions are likely to be common, and yet their contribution in shaping host phenotypic variation and adaptation are underexplored. As the study of microbiomes moves beyond descriptive attributes, experimentally assessing the interactions between host and microbial evolution are a key research priority for better understanding the interplay in host-microbiome interactions $[11,12]$.

The fruit fly, Drosophila melanogaster, has emerged as an excellent model to study host-microbe interactions. Flies have relatively simple microbiomes, often composed of fewer than 10 bacterial species from acetic acid and lactic acid bacteria families [13]. The microbiome is largely environmentally acquired through feeding, defecation, and egg smearing during oviposition $[14,15]$. After hatching, larvae acquire their microbes through a combination of microbial persistence and colonization and form associations over the lifespan of the fly [16-18]. Thus, the microbiome is mostly environmentally acquired, and these microbes also must persist independently in the habitat. Many fly-associated microbes can be individually cultured and reconstituted in 
various combinations to inoculate sterile flies $[13,19]$. With the feasibility of microbiome manipulations and rich genetic resources, Drosophila is an ideal model to study the interplay between host genetic, microbiome, and environment in shaping host phenotypes.

The microbiome shapes many different aspects of Drosophila biology. Axenic flies (without microbiomes) develop slower, display metabolic defects, and dysregulated immune systems compared to those with normal microbiomes $[13,17]$. Different components of the microbiome also affect Drosophila traits in different ways. Generally, Acetobacter species tend to accelerate development while Lactobacillus slow development [20,21]. The variation in microbiomes also affects how flies utilize and store energy, but this depends on fly genetics $[20,22]$. For example, lipid storage in only some host genotypes increased in the presence of Acetobacter tropicalis $[22,23]$. Furthermore, interactions within the microbiome can shift life history tradeoffs between lifespan and fecundity. More complex communities that include multiple species of Acetobacter and Lactobacillus developed and died faster, but offset fitness costs by increasing fecundity compared to mono-association with the same bacteria [24]. Finally, microbiome composition shaped genomic divergence in field mesocosms during seasonal evolution that matched traits associated with natural Drosophila populations [21, 25]. These studies demonstrate the range of effects associated with the microbiome, and importantly, the complex web of host genetic and microbial control that contribute to variation in fly phenotypes.

While the above studies identify key host genotype $\mathrm{x}$ microbiome interactions, missing are the environmental effects. Environmental stressors may increase the relative importance of microbial costs or benefits [26-28]. For Drosophila, the microbiome may be critical to mitigate dietary stressors, but few studies have tested the role of the microbiome in dietary adaptation. One study found that Lactobacillus evolved to promote larval growth and development in mono-association with Drosophila on nutrient poor diets [29]. However, nutrient poor diets often limit microbial growth in the fly gut [30]. More so, axenic flies adapted to nutrient poor diets had similar survival and metabolic phenotypes as conventional flies [31], suggesting no benefit of the microbiome in stressful environments. While these studies address what happens to fly or the microbiome, how host evolution and the microbiome together shape host survival in stressful environments remains poorly understood.

Here, we leveraged $\sim 150$ generations of fly and microbial adaptation to high sugar (HS) diets. HS diets exert stress on flies, and flies exhibit similar obesity-like phenotypes as humans with elevated triglycerides, insulin resistance, and shortened lifespan [32-34]. To cope with HS stress, flies must convert sugar into lipids [35]. One genus of the microbiome, Acetobacter consumes and reduces sugar in the fly gut [36], suggesting Acetobacter may contribute to fly adaptation to HS diets. To test this hypothesis, we performed a fully reciprocal host $\mathrm{x}$ microbiome $\mathrm{x}$ diet transplant experiment using the evolved HS fly genotype and HS Acetobacter. If the microbiome facilitates fly adaptation in HS diets, then transferring the HS microbiome into naive hosts may confer adaptive traits. Our results highlight the complexities of environmental context dependency on host-microbiome interactions.

\section{METHODS}

\section{Fly populations}

All fly populations were maintained at $25^{\circ} \mathrm{C}$ with 12 hour light:dark cycles. The base population consisted of an outbred population created by crossing 15 inbred lines initially collected in the Netherland (a subset of the Global Diversity Lines) [37]. Briefly, we crossed each inbred line to another using a round-robin design. The resulting F1s were crossed in a similar pattern to ensure parental representation and that no chromosome would be lost in the first round of crosses. The resulting F2 individuals were placed in large population cages, maintained at large population size (>10,000 flies) and allowed to interbreed freely for $\sim 50$ generations before high sugar (HS) selection began. Flies were maintained on the control diet composed of $10 \%$ glucose, $10 \%$ yeast, $1.2 \%$ agar, with $0.04 \%$ phosphoric acid and $0.4 \%$ propionic acid as preservatives. Three replicate populations for HS selection were derived from the base population and maintained on the same diet, but with an additional 13\% sucrose and at lower population size ( $>5000$ flies/cage until generation 100 and $\sim 2000$ flies/cage after). The approach used here allowed for natural selection on HS diets; we did not guide or select for particular traits as is often used in experimental evolution in Drosophila. In parallel, three replicate populations were reared on control diets as is standard for experimental evolution studies, but these are not the focus of this study. Because we were interested in the microbiome transferring adaptive traits to naive hosts, we defined the outbred base population as our control, non-evolved population. We consider the two populations, $\mathrm{C}$ and HS, to be two different genotypes. We note that the flies are not traditional inbred lines and still retain genetic diversity, but for the purposes of this study, we consider their evolutionary history to have generated two distinct genotypes.

\section{Transplanting microbiomes to test for adaptive significance}

To test if the microbiome can transfer adaptive traits, we performed a reciprocal microbiome transplant experiment (Fig. 1). All host genotypes $\mathrm{x}$ microbiome $\mathrm{x}$ diet interactions were generated in the reciprocal transplant. Both genotypes (C and HS flies) were inoculated with microbiomes (C, HS) or kept sterile and raised in both diets (C and HS) for a total of 12 treatments. This experimental design allowed us to evaluate 


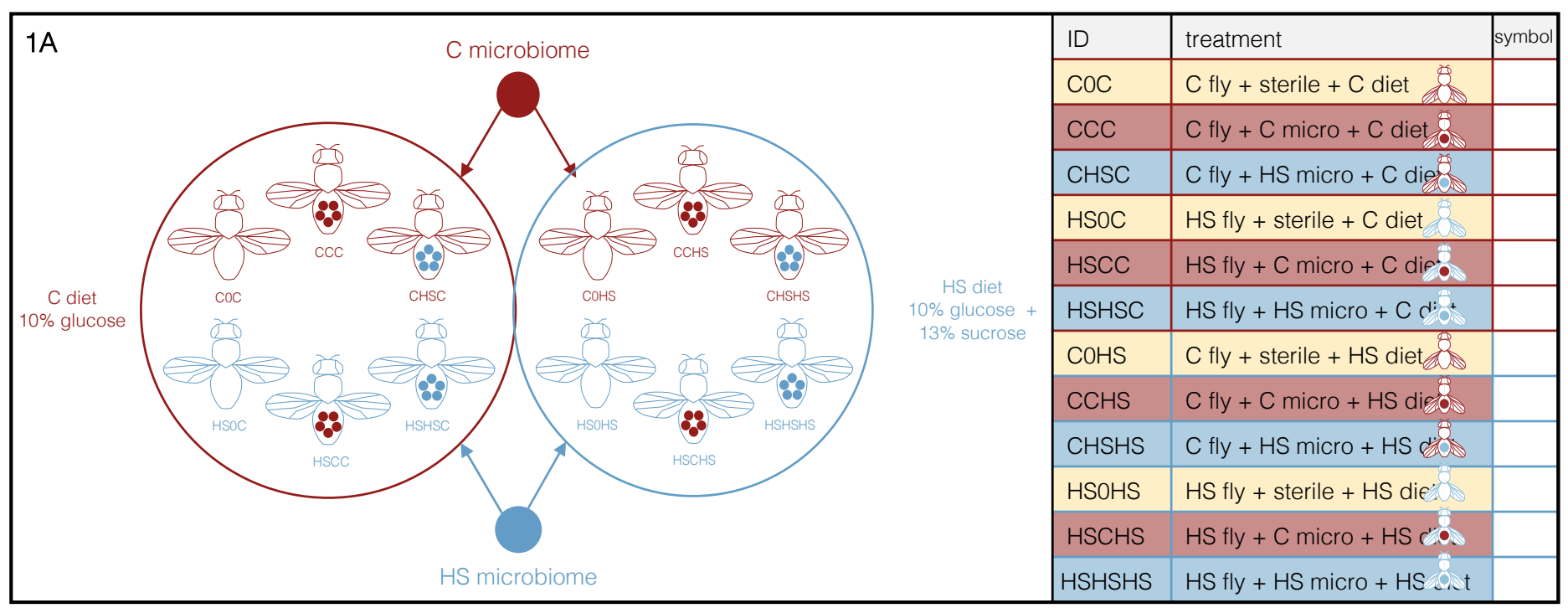

Fig. 1: We leveraged $>150$ generations of fly adaptation to HS diets to study the role of the microbiome in adaptation.

A) Experimental design for fully reciprocal host $x$ microbiome $x$ diet transplant to test the role of microbial evolution to fly adaptation. Fly genotypes (C or HS) were inoculated with microbes (none, $\mathrm{C}$, or HS) in two diets ( $\mathrm{C}$ or HS). The same experimental design was used in two iterations: community transplant or Acetobacter transplant. The table shows the abbreviated treatment codes used throughout this study, and it is colored to emphasize the microbiome treatment. The symbols represent the same information visually diagrammed as flies. Red colors represent control, blue colors represent high sugar, and yellow (or empty flies) represent sterile treatments.

how host $\mathrm{x}$ microbiome $\mathrm{x}$ environment interactions influence the transfer of adaptive phenotypes.

We performed an initial microbiome transplant using fly frass, and we detailed the methods and results in the supplement. In brief, we found that diet strongly structured the microbiome of both donor and recipient flies (Supp. Fig. 1), affected developmental timing and fecundity (Supp. Fig. 2), but the recipient microbiome did not strongly resemble the donor, suggesting the transplant was not faithful. To better understand the host-microbiome interaction, we identified the differentially abundant bacteria between $\mathrm{C}$ and HS flies through ANCOM (analysis of composition of microbiomes) implemented in QIIME2 [38]. For ANCOM, analysis was performed on ASVs (amplicon sequence variants). Only ASVs present in $>5$ fly samples were included. We cultured the most differentially abundant bacteria from HS flies, $A$.

pasteurianus, and the significantly differentially abundant bacteria within the Acetobacter genus and enriched in C flies, A. indonesiensis. We confirmed 16S rRNA sequence identity using Sanger sequencing. Because the 16S rRNA phylogeny is discordant with a multilocus phylogeny in the

Acetobacteraceae [39] and for simplicity, we refer to $A$. indonesiensis as $\mathrm{C}$ Acetobacter and A. pasteurianus as $\mathrm{HS}$ Acetobacter.

To perform the transplant, Acetobacter was cultured in liquid MRS media at $25^{\circ} \mathrm{C}$ for 2-3 days. Bacterial density was normalized to $\mathrm{OD}_{600}=0.1$, and $50 \mu 1$ of bacteria (or PBS for sterile treatments) was used to inoculate axenic eggs in sterile diets [19]. For each treatment, 6-10 replicates were maintained. Flies were then reared in a biosafety cabinet at $25^{\circ} \mathrm{C}$ and 12:12 light:dark cycle through the lifespan of the recipient flies. At 8-10 days post eclosion, a subset of 4-6 flies from all replicates were homogenized in $100 \mu \mathrm{PBS}$ and plated on MRS media. Bacterial growth was visually confirmed to be the original Acetobacter isolates and sterility for sterile treatments.

\section{Phenotypic effects of the Acetobacter transplant}

To measure developmental time, we counted the number of new pupae every 6 hours during their daytime (i.e., $8 \mathrm{am}, 2 \mathrm{pm}, 8 \mathrm{pm}$ ) until all treatments began eclosing. We used the survival [40] and survminer [41] packages in R to analyze development data. We used Cox proportional hazards to identify how interactions between fly genotype, microbiome, and diet influence development time with the following model:

$$
\begin{aligned}
& \mathrm{DT}=\mathrm{y}+\beta_{1} \mathrm{G}+\beta_{2} \mathrm{M}+\beta_{3} \mathrm{E}+\beta_{4}\left(\mathrm{G}^{*} \mathrm{M}\right)+\beta_{5}\left(\mathrm{M}^{*} \mathrm{E}\right)+\beta_{6}\left(\mathrm{G}^{*} \mathrm{E}\right)+ \\
& \beta_{7}\left(\mathrm{G}^{*} \mathrm{M}^{*} \mathrm{E}\right)+\mathrm{e}
\end{aligned}
$$

Where DT $=$ developmental time (pupation), $\mathrm{y}=$ grand mean, $\mathrm{G}=$ fly genotype (C or HS), $\mathrm{M}=$ Acetobacter treatment $(\mathrm{C}$, $\mathrm{HS}$, or sterile $), \mathrm{E}=\operatorname{diet}(\mathrm{C}$ or HS$)$, and $\mathrm{e} \sim \mathrm{N}\left(0, \sigma_{\mathrm{e}}\right)$ as residual error.

For fecundity, we counted the number of eggs laid by individual 8-10 day old females $(\mathrm{N}=644)$. Females were placed in a 24-well plate with C and HS oviposition media. Oviposition media was similar to the fly diets, but with yeast extract to make the media transparent for ease of egg counting. 
We did not find a difference in egg counts between $\mathrm{C}$ and $\mathrm{HS}$ oviposition media (Supp. Fig. 3, Kruskall-Wallis $\mathrm{X}^{2}=2.85$, $\mathrm{df}=1, \mathrm{p}=0.09$ ), and for the subsequent analyses, pooled together egg counts from both oviposition media. To minimize plate effects, two treatments were assayed on each plate, and treatments were randomly assigned across plates. Females laid eggs for $\sim 4-6 \mathrm{hrs}$ at $25^{\circ} \mathrm{C}$ during the late afternoon ( $\left.2 \mathrm{pm}-8 \mathrm{pm}\right)$ to capture similar circadian rhythms in egg laying across all treatments. We analyzed fecundity using a zero-inflated Poisson distribution implemented in glmmTMB [42] with the following model:

$$
\mathrm{F}=\mathrm{y}+\beta_{1} \mathrm{G}+\beta_{2} \mathrm{M}+\beta_{3} \mathrm{E}+\beta_{4}\left(\mathrm{G}^{*} \mathrm{M}\right)+\beta_{5}\left(\mathrm{M}^{*} \mathrm{E}\right)+\beta_{6}\left(\mathrm{G}^{*} \mathrm{E}\right)+
$$$$
\beta_{7}\left(G^{*} M^{*} E\right)+d+e
$$

Where $\mathrm{F}=$ egg count from individual females, $\mathrm{y}=$ grand mean, $\mathrm{G}=$ fly genotype $(\mathrm{C}$ or $\mathrm{HS}), \mathrm{M}=$ Acetobacter treatment (C, HS, or sterile), $\mathrm{E}=\operatorname{diet}\left(\mathrm{C}\right.$ or HS), where $\mathrm{d} \sim \mathrm{N}\left(0, \sigma_{\mathrm{o}}\right)=$ random effect of oviposition plate ( $\mathrm{N}=29$ plates), and $\mathrm{e} \sim \mathrm{N}(0$, $\left.\sigma_{e}\right)$ as residual error. We tested for significance of fixed effects using Type III ANOVA with Kenward-Roger degrees of freedom using the car package in $R$ [43]. Least square means were used to visualize the interactions with the emmeans package in R [44].

For nutritional indices, we assayed pools $(\mathrm{N}=95)$ of three age-matched (8-10 days old) females for protein and total triglycerides. Protein serves as a proxy for size and was measured using a Bradford assay (BioRad 5000205). Total triglycerides represent fat stores for flies and assayed using a colorimetric assay (Sigma TR0100-1KT). Triglycerides were normalized by protein amount [45]. Each assay was performed in triplicate in 96 well plates with all 12 treatments in each plate to minimize batch effects. Triplicate values were averaged; values with standard deviation $>0.02$ were removed from analysis. The replicates of treatments were assayed across different assay plates. We visualized the protein and triglyceride data by regressing out the effect of the assay plates to show mean-centered values. We modeled the response as:

$\mathrm{Y}=\mathrm{y}+\beta_{1} \mathrm{G}+\beta_{2} \mathrm{M}+\beta_{3} \mathrm{E}+\beta_{4}\left(\mathrm{G}^{*} \mathrm{M}\right)+\beta_{5}\left(\mathrm{M}^{*} \mathrm{E}\right)+\beta_{6}\left(\mathrm{G}^{*} \mathrm{E}\right)+$ $\beta 7\left(G^{*} M^{*} E\right)+d+e$

Where $\mathrm{Y}=$ protein or triglyceride amount, $\mathrm{y}=$ grand mean, $\mathrm{G}$ $=$ fly genotype $(\mathrm{C}$ or $\mathrm{HS}), \mathrm{M}=$ Acetobacter treatment $(\mathrm{C}, \mathrm{HS}$, or sterile), $\mathrm{E}=\operatorname{diet}(\mathrm{C}$ or HS$)$, where $\mathrm{d} \sim \mathrm{N}\left(0, \sigma_{0}\right)=$ random effect of assay plate ( $\mathrm{N}=14$ assay plates), and $\mathrm{e} \sim \mathrm{N}\left(0, \sigma_{e}\right)$ as residual error. The three way interaction between fly genotype, Acetobacter, and diet was not significant and was removed from the model. We tested for significance of fixed effects using Type III ANOVA with Kenward-Roger degrees of freedom using the car package [43]. Least square means were visualized to show interaction effects with the emmeans package in $\mathrm{R}$ [44].

\section{RESULTS}

Fly microbiome responds to $H S$ selection

Here, we leveraged $\sim 150$ generations of fly and microbial adaptation to HS diets to understand how the microbiome can contribute to host evolution. First, we established that the microbiome changed between $\mathrm{C}$ and $\mathrm{HS}$ flies (Fig. 2A). HS flies were dominated by Acetobacter pasteurianus $(86.7 \%+/-1.8 \mathrm{SE})$ and unclassified Acetobacteraceae strain $(5.1 \%+/-1.8 \mathrm{SE})$, while $\mathrm{C}$ flies were more diverse with Algoriella xinjiangensis $(37.3 \%+/-5.3 \mathrm{SE})$, Acetobacter persici ( $16.9 \%+/-2.6 \mathrm{SE})$, and Acetobacter indonesiensis $(14.8 \%+/-2.1 \mathrm{SE})$. The most differentially abundant bacteria were $A$. pasteurianus and Acetobacteraceae strain for the HS flies, while $A$. xinjiangensis and $A$. indonesiensis were associated with C flies (Fig. 2B, Supp. Table 1). These bacteria are unique to either $\mathrm{C}$ or HS flies, demonstrating that the HS diet selected for different fly microbiomes.

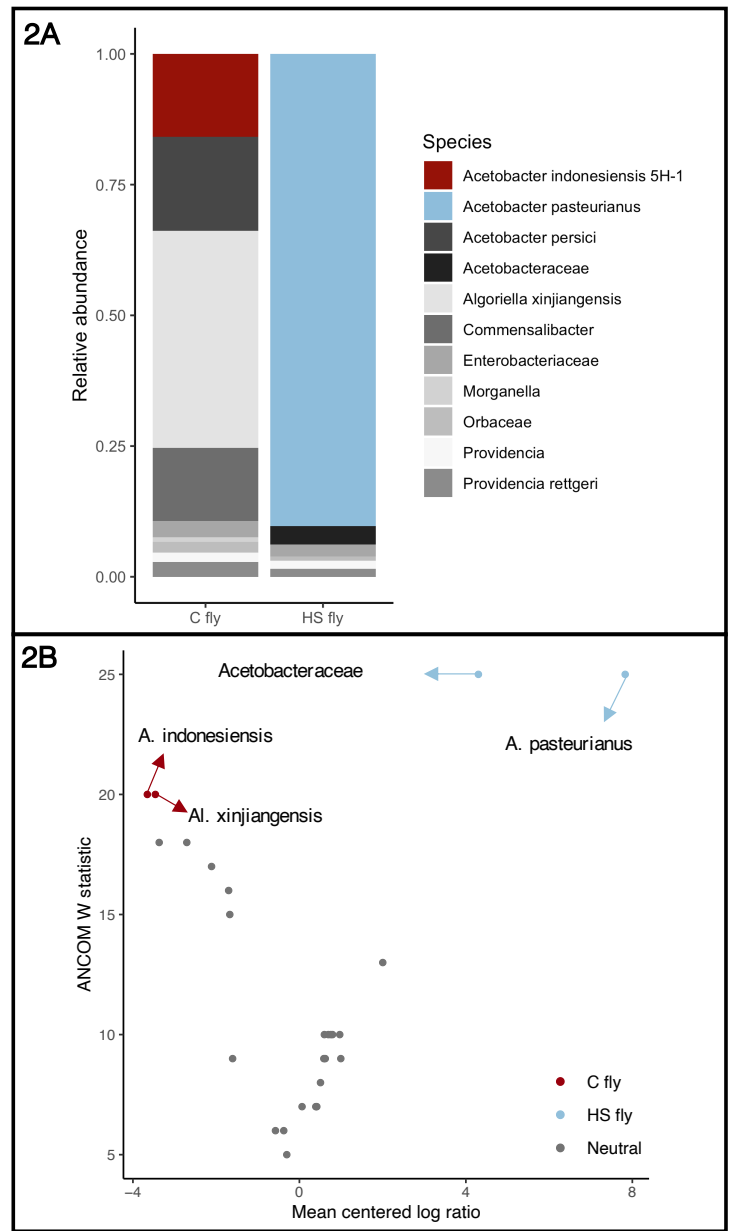

Fig. 2: The microbiome in HS flies is different from $C$ flies. A) Relative abundance of microbiome determined using 16S rRNA sequencing. The $C$ fly microbiome is enriched for $A$. indonesiensis, $A$. persici, and Algoriella xinjiangensis. The HS fly microbiome is enriched for $A$. pasteurianus. B) ANCOM results for differentially abundant bacteria between $\mathrm{C}$ and $\mathrm{HS}$ flies. Colored points represent significantly different ASVs and arrows denote taxonomic classification. 

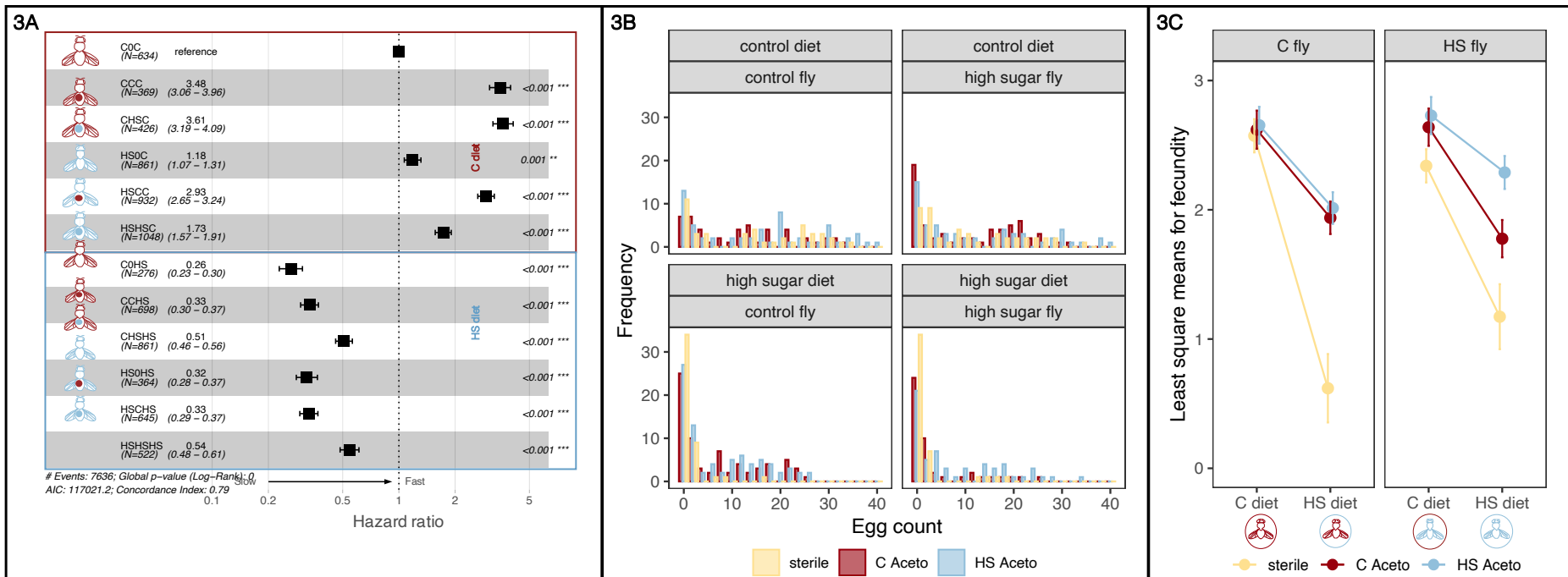

Fig. 3: Life history effects of the Acetobacter transplant.

A) Hazard ratio plot for Acetobacter effects on development time. Boxplots show hazard ratios and 95\% confidence intervals for each treatment relative to $\mathrm{COC}$. Mismatches between diet and microbiome slowed development. In the $\mathrm{C}$ diet, HS flies with $\mathrm{C}$ Acetobacter developed faster than HS flies with HS Acetobacter. However, on the HS diet, C Acetobacter slowed, while HS Acetobacter accelerated development. The interaction effects on the Cox proportional hazards model between fly genotype $x$ Acetobacter $x$ diet best explained variation in development $\left(\right.$ log-likelihood= $-58500, \mathrm{df}=7, \mathrm{C}^{2}=465.8$, $\mathrm{p}<0.001)$. B) Histogram of fecundity from individual females in the Acetobacter transplant, faceted by fly genotype and diet. Colors represent the Acetobacter treatment. Fecundity is zero skewed, especially for flies on HS diets. C) Least square means (LSM) from fecundity, modeled with a zeroinflated Poisson distribution, show fly genotype $\mathrm{x}$ Acetobacter $\mathrm{x}$ diet interactions shaped fecundity. Colors represent Acetobacter treatment. Error bars show standard error for LSMs. For the HS fly genotype, the HS Acetobacter increased fecundity relative to the C Acetobacter, suggesting a fecundity benefit with the adapted microbiome.

\section{Transplanting Acetobacter influences life history traits}

To test if transferring the microbiome could transfer adaptive phenotypes to naive hosts, we performed a fully reciprocal, host genotype $\mathrm{x}$ microbiome $\mathrm{x}$ diet transplant experiment. We first performed a fecal transplant in flies, but found that the transplant was not faithful (Supp. Fig. 1), as is common for fecal transplants in other taxa [46-48]. We then transplanted the dominant Acetobacters from $\mathrm{C}$ and HS diets identified above in a fully reciprocal experiment and measured life history and metabolic traits.

Development was shaped by interactions between fly genotype, Acetobacter, and diet as was observed in the whole community transplant (Fig. 3A, Supp. Table 2). Importantly, like in the microbiome transplant, all sterile treatments developed slowest. However, flies responded differently to the Acetobacter transplant. On the $\mathrm{C}$ diet, fly genotype influenced the response to the Acetobacter transplant. HS flies with the C Acetobacter developed more rapidly (avg. $140.8 \mathrm{hrs}+/-0.54$

$\mathrm{SE}$ ) than with the HS Acetobacter (avg. $147.6 \mathrm{hrs}+/-0.60$

$\mathrm{SE})$. There was no effect for the $\mathrm{C}$ fly. However, on the HS diet, the HS Acetobacter accelerated development (HS Acetobacter avg. $176.5 \mathrm{hrs}+/-.50 \mathrm{SE}, \mathrm{C}$ Acetobacter avg. $186.8 \mathrm{hrs}+/-0.60 \mathrm{SE})$. The three-way interaction between fly genotype, Acetobacter, and diet significantly affected development time (Supp. Table 2, log-likelihood $=-58500, \mathrm{X}^{2}$ $=47.625, \mathrm{p}<0.0001)$.
Fecundity responded to interactions between fly genotype, Acetobacter, and diet (Fig. 3B, Supp. Table 3). On average, treatments were more fecund on the $\mathrm{C}$ diet, while treatments on HS diets laid 54.3\% fewer eggs (C avg. 13.0 eggs +/-0.6 SE, HS, avg. 5.9 eggs +/- 0.4 SE). Sterile treatments substantially lowered fecundity, only on the HS $\operatorname{diet}(+$ microbes avg. 7.45 eggs $+/-0.5 \mathrm{SE}$, sterile avg. 1.67 eggs +/- 0.4 SE). Notably, the response to Acetobacter treatment depended on fly genotype for the HS diet. On the HS diet, HS flies with HS Acetobacter (avg. 8.4 eggs +/-1.4 $\mathrm{SE}$ ) were more fecund than with C Acetobacter (avg. 4.5 eggs $+/-1.0 \mathrm{SE}$ ). The interaction between fly genotype, microbiome, and diet significantly affected fecundity (interaction $\mathrm{X}^{2}=16.4, \mathrm{df}=2, \mathrm{p}=0.0003$, Fig. 3C, Supp. Table $3)$.

To gain insight into how these interactions shape host metabolism, we assayed flies for protein and triglyceride (TAG) levels. Protein levels were primarily shaped by interactions between fly genotype and diet (Fig. 4A, Supp. Table 4). On the C diet, HS flies had higher protein levels than $\mathrm{C}$ flies, but this relationship was not observed on the HS diet (Fig. 4B, fly genotype $\mathrm{x}$ diet interaction, $\mathrm{F}_{2,78.1}=18.3, \mathrm{p}<$ 0.0001). Acetobacter modified this interaction, but also only on the $\mathrm{C}$ diet (Acetobacter $\mathrm{x}$ diet interaction, $\mathrm{F}_{2,78.2}=3.26, \mathrm{p}=$ $0.04)$. TAG levels were also shaped by interactions between fly genotype and diet (Fig. 4C, Supp. Table 5). On the C diet, 
Henry et al., 09-17-2020 bioRxiv

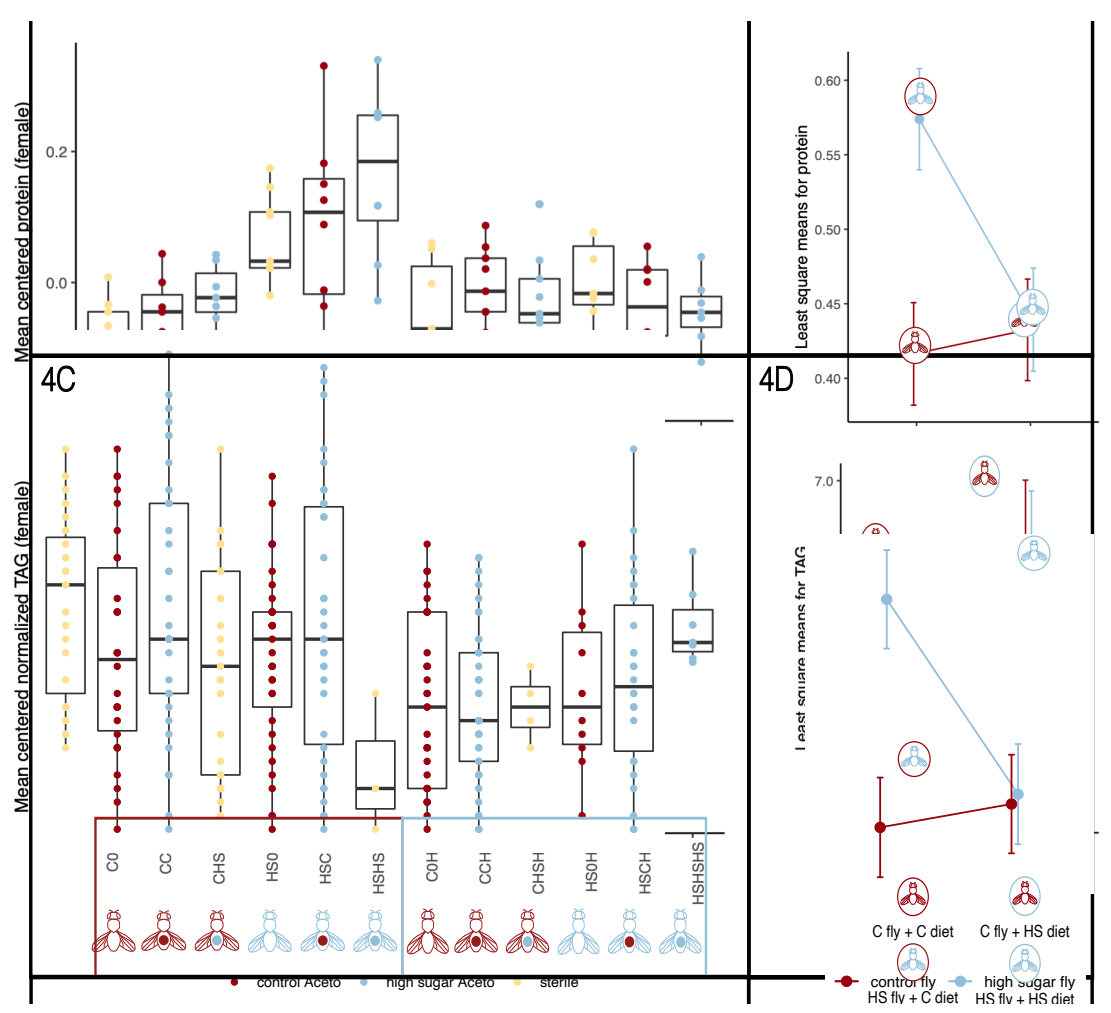

Fig. 4: Metabolic effects of the Acetobacter transplant.

A) Boxplot for protein levels. Protein amount is a proxy for size in Drosophila. Each point represents a pool of three age-matched female flies. Protein values were mean centered for each batch assayed. Fly genotype, diet, and their interaction significantly affected size $\left(F_{1,78.1}=18.3\right.$, $\left.p<0.0001\right)$, while Acetobacter had minimal effects. B) Least square means (LSM) from the mixed model shows significant interactions between fly genotype and diet. Error bars show standard error for LSMs. For HS flies, protein levels responded to diet, but C flies did not significantly differ. C) Boxplot for triglycerides (TAG) normalized to protein. TAG amount reflects fat storage in Drosophila. Each point represents a pool of three age-matched female flies. TAG levels were mean centered to control for each batch assayed. Like for protein levels, interactions between fly genotype and diet shaped TAG levels $\left(F_{1,78.1}=\right.$ 9.00, $p=0.004$ ), but Acetobacter had no effect. D) LSMs from the mixed model shows the significant interaction between fly genotype and diet. Error bars show standard error for LSMs. Like for protein, TAG levels responded to diet for HS flies, but not for C flies.

HS flies had lower TAG levels than C flies, but this relationship was not observed on the HS diet (Fig. 4D, fly genotype $\mathrm{x}$ diet interaction, $\mathrm{F}_{1,78.0}=9.00, \mathrm{p}=0.004$ ). Acetobacter, alone or through interactions, did not influence TAG levels. Thus, fly metabolism was primarily shaped by host genetics and diet, but not by differences between Acetobacters.

\section{DISCUSSION}

Here, we explored how the microbiome influences host fitness in stressful environments. Through reciprocal host $\mathrm{x}$ microbiome $\mathrm{x}$ diet transplant experiments, we show that $\mathrm{G}_{\mathrm{H}}$ x GM X E interactions shape host fitness by modifying developmental timing and fecundity. Our results highlight the complex interactions generated by the microbiome, and we discuss important future directions to further dissect these relationships.

\section{Acetobacter dominates HS microbiome}

Acetobacter was the most abundant genus in the HS flies (Fig. 2). Acetobacter are common in flies, both in laboratory settings and wild environments [17, 49-52]. Furthermore, Acetobacter are sugar specialists and tolerate highly acidic environments, like during vinegar fermentation
[53]. In the context of Drosophila biology, some strains of Acetobacter reduce glucose levels and enhance oxidation of ethanol, which promotes larval development $[54,55]$. Additionally, reducing glucose levels is critical for flies to survive HS diets [35]. Over the course of experimental evolution, both fly and microbiome were exposed to HS diets, and in turn, the HS microbiome was dominated by $A$.

pasteurianus (Fig. 2B). Taken together, these results indicate that HS selection also changed the fly microbiome, selecting for bacteria that could survive HS stress.

In our attempt to transplant the microbiome, we expected that the HS diet would promote differentiation between $\mathrm{C}$ and HS microbiome recipients. However, several lines of evidence suggest that the microbiome transplant was not faithful as recipient microbiomes did not resemble donor microbiomes. First, both the $\mathrm{C}$ and HS microbiomes were distinct in bacterial composition and variance across donors, frass, and recipients (Supp. Fig. 4). Only a few species were common in both fly and frass, but were in different relative abundances, much like is found in mammals and their fecal microbiomes [56, 57]. More so, the amount of variation in microbial community composition explained by the microbiome transplant was much lower on the HS diet (HS: 
$r^{2}=0.04, C: r^{2}=0.43$, Supp. Fig. 1). While we did not detect contamination in the sterile treatments (Supp. Fig. 5), we are unsure why the microbiome transplant was unfaithful. This appears to be a common issue with fecal transplants in mammals as well [46-48]. We did note that the abundance of microbes differed between $\mathrm{C}$ and HS frass in the transplant (Supp. Fig. 6). This suggests that the HS diet also suppresses microbial abundance, in addition to microbial diversity. Much remains unknown about how ecological and evolutionary conditions can promote or impede microbiome transplants [46-48]. Host and environmental factors that support microbial diversity may increase the success of microbiome transplant [58, 59], and those factors that limit microbial growth impede transplants, much like the HS diet that limited both microbial diversity and growth. Because of the difficulty in interpreting the low fidelity of the microbiome transplant and the dominance in the HS microbiome by a single Acetobacter species, we subsequently focused on the phenotypic effects of the two dominant Acetobacters in the $\mathrm{C}$ and HS microbiomes.

\section{Host $x$ microbiome $x$ environment interactions shape life history traits}

In Drosophila, as well as other holometabolous insects, life history traits represent a classic tradeoff between survival and reproduction [60]. Faster development increases fitness because it maximizes reproductive events before death. Furthermore, Drosophila evolved in patchy, ephemeral habitats that enhance competition among larvae and predation risk [61, 62]; faster development can reduce these risks. Delayed development imposes fitness costs unless fecundity later in the host lifespan compensates for that delay. The microbiome has emerged as a novel factor that frequently shapes life history traits of developmental time and fecundity in Drosophila [20, 24, 54, 63]--though all of these studies were in benign environments. Our results advance these findings by investigating the fly response in a stressful environment and the role of host evolution in mediating this relationship.

For both developmental time and fecundity, mismatches between host evolution, microbiome, and diet led to fitness costs. For developmental timing, HS flies with HS Acetobacter delayed development on the $\mathrm{C}$ diet, but for both fly genotypes, the HS Acetobacter accelerated development on the HS diet (Fig. 3A). The development effects suggest fitness benefits for the HS Acetobacter only on the HS diet, but costs for the $\mathrm{C}$ Acetobacter. Fecundity also suffered from mismatches with the environment, though predominantly for HS flies on the HS diet (Fig. 3C). HS flies experienced relative increases in fecundity with the HS Acetobacter, but decreased with the $\mathrm{C}$ Acetobacter. $\mathrm{C}$ flies did not respond to differences between the $\mathrm{C}$ and HS Acetobacter in either diet. The $\mathrm{G}_{H} \times \mathrm{G}_{\mathrm{M}} \times \mathrm{E}$ interactions for both development and fecundity suggest that the $\mathrm{C}$ and HS flies evolved to respond differently to microbial variation, especially in the stressful HS diet.

Our results highlight the surprising responsiveness of developmental timing to microbial variation in Drosophila. More so, recent studies have suggested that microbial variation often modulates developmental timing in many different kinds of organisms, like killifish [64], dung beetles [65], and Arabidopsis [66]. One proposed mechanism is that the microbiome triggers development that matches host phenotypes to maximize fitness in the local environment [67]. If so, the microbes must provide a more reliable signal than the host can detect alone, but the host must also have a mechanism to detect the signal.

Our results suggest that HS Acetobacter may provide a signal to shift development on the HS diet, potentially through promoting fat storage in larvae as found in other Acetobacter species [54, 55]. For signal detection, the responsiveness of the HS fly to Acetobacter variation suggests the evolution of a novel mechanism to increase fecundity through the insulin/insulin-like signaling (IIS) pathway. IIS modulates endogenous developmental timing and reproduction, but is also affected by the microbiome $[54,68$, 69]. IIS is also directly involved in mitigating HS stress as flies with a deficient IIS pathway exhibit slower development and shortened lifespan on HS diets [32]. Thus, the IIS pathway may integrate signals from the microbiome. While we did not detect Acetobacter effects on two metabolic indices (Fig. 4), additional phenotyping for other indices, like glucose or trehalose, and in the larvae may provide additional insight into the mechanistic basis underlying the life history phenotypes. Identifying the molecular variation and gene expression patterns that differ between C and HS flies, like the IIS pathway, will demonstrate how the microbiome altered the response to selection in hosts during adaptation.

\section{Adaptive potential of the microbiome depends on evolutionary and ecological context \\ Many have predicted that the microbiome could accelerate local adaptation in the host, facilitating phenotypic changes to buffer local stressors [6, 70-72]. If so, transplanting the locally adaptive microbiomes should confer adaptive phenotypes to naive individuals. However, the benefit may depend on interactions between host genotype and environment. Gн х Gм $\mathrm{x}$ E interactions have the potential to facilitate or impede evolution in challenging environments $[11,12]$. Through combining experimental evolution with reciprocal transplants, our data allowed us to test if and how ecological context and host evolution shape the adaptive potential of the microbiome.}

To shape adaptive potential, the microbiome must contribute to host phenotype, not unlike traditional (host) additive genetic variance [72]. For development, the microbiome conferred an adaptive phenotype--HS Acetobacter 
accelerated development similarly for both the naive $\mathrm{C}$ and adapted HS fly in the HS diet (Fig. 3A). The $\mathrm{G}_{\mathrm{H}} \mathrm{x} \mathrm{G}_{\mathrm{M}} \mathrm{x}$ E interaction for development illustrates how diet, the ecological context, may increase the fitness benefit of locally adaptive microbiomes. However, the $\mathrm{G}_{\mathrm{H}} \mathrm{x} \mathrm{G}_{\mathrm{M}} \mathrm{X} \mathrm{E}$ interaction that shaped fecundity is suggestive of how genetic interactions may impede the transfer of adaptive potential. The naive host, $\mathrm{C}$ flies, did not receive a fecundity increase from the HS Acetobacter in the HS diet, but HS flies did (Fig. 3C). The presence of $\mathrm{G}_{\mathrm{H}} \times \mathrm{G}_{\mathrm{M}} \times \mathrm{E}$ interactions does not imply that the microbiome was unimportant to fly evolution. Rather, the responsiveness of the HS fly to microbial variation in both diets suggests that HS flies evolved a specialized association with their microbiome. Thus, the HS fly evolved to select for beneficial Acetobacter traits, which may lead to the dominant HS Acetobacter. Taken together, our results demonstrate how both the ecological context and host evolution shape the adaptive potential of the microbiome.

More broadly, the $\mathrm{G}_{\mathrm{H}} \mathrm{x} \mathrm{G}_{\mathrm{M}} \mathrm{x}$ E interactions observed here provide several insights into host-microbiome evolution. First, our work contributes to the growing body of literature that environmentally acquired microbes are important in stressful environments. Stressful environments are predicted to increase the relative importance of mutualisms [26-28], though how often the microbiome behaves evolutionarily like a traditional mutualism remains contentious $[4,5,72]$. In plants, stress-adapted soil microbiomes improve germination and seedling survival in stressful environments [73, 74], highlighting the importance of early life traits like we also observed for development. Second, host genotypes may vary in their ability to leverage beneficial microbes in stressful environments. Genetic interactions may limit the benefits of locally adaptive microbiomes, like we observed for fecundity. Understanding these $\mathrm{G}_{\mathrm{H}} \mathrm{x} \mathrm{G}_{\mathrm{M}}$ interactions will be especially important in using evolution to engineer beneficial microbiomes to solve pressing problems in public health and agriculture $[11,12,75]$. Our results suggest how the microbiome may shape host adaptation, but we focused only on the endpoints of fly adaptation. Longitudinal sampling over the evolutionary path will provide key insights into how host genetics and environment shape the adaptive potential of the microbiome.

Finally, we acknowledge that despite the $\sim 150$ generations of adaptation to the HS diet, the HS diet still comes at a fitness cost to the HS flies. For example, developmental time was still slower on the HS diet compared to the $\mathrm{C}$ diet (Fig. 3A). HS flies were less fecund on the HS diet than on the $\mathrm{C}$ diet (Fig. 3C). For metabolic indices, genotypic differences were only observed on the $\mathrm{C}$ diet (Fig 4). One possibility for these unexpected results is the use of dechorionation to manipulate the microbiome.

Dechorionation, which removes the chorion that protects eggs, is a common tool for the Drosophila microbiome [13, 19]. Dechorionation has conflicting effects on different aspects of fly physiology. One study found no significant effect on metabolic indices, like protein and TAG levels, compared to conventionally reared flies [76]. However, another study found that dechorionation increased overall mortality, though improved starvation resistance [77]. It is possible that dechorionation affected our results, but we did not test for this effect. We raise this point as one challenging aspect of using experimentally evolved hosts for microbiome manipulations, as adaptation to novel environments may change host biology in unexpected ways.

\section{Conclusions and research priorities}

Here, we leveraged host and microbial evolution from $\sim 150$ generations of adaptation to a stressful diet. The fly populations used in this experiment were originally conceived as an Evolve \& Resequence (E\&R) experiment. The E\&R approach in Drosophila uses outbred populations to identify signatures of selection across the genome and has illuminated many important findings about the role of demography, population genetics, and genetic architecture in shaping adaptive traits $[78,79]$. However, missing from this framework is the role of the microbiome. In a survey of ten E\&R experiments, the microbiome also frequently responded to experimental evolution, particularly in traits associated with metabolism [80]. Taken together, our data here also highlight how the microbiome may contribute to host adaptation, previously unseen by focusing only on the Drosophila genome. Our study provides the first experimental dissection of the microbial response and influence on host traits in the E\&R context. Through reciprocal transplants, we identified how interactions between fly genotype, microbiome, and diet shape fitness related traits.

The data here suggest that the microbiome can contribute to host fitness, but many questions remain about its evolutionary impact. We focused on the endpoints of evolution between host and microbiome, but the evolutionary trajectory of the interaction is unknown. Longitudinal studies that characterize microbial change as well as phenotypic effects would demonstrate if microbial evolution precedes host evolution and whether this shapes the host evolutionary trajectory. To better understand the evolutionary trajectory, reciprocal transplants over the course of adaptation will also show the relative importance of the microbiome compared to host evolution. Experimental evolution is a powerful, but underutilized tool in the study of host-microbiome evolution $[11,72,81]$. Ultimately, the evolutionary benefits of the microbiome will depend on both the environmental and host genetic context, as suggested by the complex interactions observed here. Future experiments that incorporate both host and microbial evolution will identify how genetic interactions shape adaptive responses in stressful environments. 


\section{Acknowledgements}

We thank members of the Ayroles lab for helpful feedback on this work. LPH was supported by NSF-GRFP under grant DGE1656466 and National Institutes of Health (NIH) grant GM124881 to JFA.

\section{REFERENCES}

1. Sommer F, Bäckhed F. The gut microbiota-masters of host development and physiology. Nat Rev Microbiol 2013; 11: 227.

2. Selosse M-A, Bessis A, Pozo MJ. Microbial priming of plant and animal immunity: symbionts as developmental signals. Trends Microbiol 2014; 22: 607-613.

3. Archie EA, Tung J. Social behavior and the microbiome. Current Opinion in Behavioral Sciences 2015; 6: 28-34.

4. Moran NA, Sloan DB. The hologenome concept: Helpful or hollow? PLoS Biol 2015; 13: e1002311.

5. Koskella B, Hall LJ, Metcalf CJE. The microbiome beyond the horizon of ecological and evolutionary theory. Nat Ecol Evol 2017; 1: 1606-1615.

6. Sudakaran S, Kost C, Kaltenpoth M. Symbiont Acquisition and Replacement as a Source of Ecological Innovation. Trends Microbiol 2017; 25: 375-390.

7. Douglas AE, Werren JH. Holes in the Hologenome: Why Host-Microbe Symbioses Are Not Holobionts. MBio 2016; 7: e02099.

8. Kikuchi Y, Hayatsu M, Hosokawa T, Nagayama A, Tago K, Fukatsu T. Symbiont-mediated insecticide resistance. Proc Natl Acad Sci U S A 2012; 109: 8618-8622.

9. Kohl KD, Dearing MD. The Woodrat Gut Microbiota as an Experimental System for Understanding Microbial Metabolism of Dietary Toxins. Front Microbiol 2016; 7: 1165.

10. Lau JA, Lennon JT. Rapid responses of soil microorganisms improve plant fitness in novel environments. Proc Natl Acad Sci U S A 2012; 109: 14058-14062.

11. Mueller UG, Sachs JL. Engineering Microbiomes to Improve Plant and Animal Health. Trends Microbiol 2015; 23: 606-617.

12. Busby PE, Soman C, Wagner MR, Friesen ML, Kremer $\mathrm{J}$, Bennett A, et al. Research priorities for harnessing plant microbiomes in sustainable agriculture. PLoS Biol 2017; 15: e2001793.

13. Douglas AE. The Drosophila model for microbiome research. Lab Anim 2018; 47: 157-164.

14. Wong AC-N, Luo Y, Jing X, Franzenburg S, Bost A, Douglas AE. The host as the driver of the microbiota in the gut and external environment of Drosophila melanogaster. Appl Environ Microbiol 2015; 81: 62326240 .

15. Blum JE, Fischer CN, Miles J, Handelsman J. Frequent replenishment sustains the beneficial microbiome of Drosophila melanogaster. MBio 2013; 4: e00860-13.

16. Bakula $\mathrm{M}$. The persistence of a microbial flora during postembryogenesis of Drosophila melanogaster. $J$ Invertebr Pathol 1969; 14: 365-374.

17. Broderick NA, Lemaitre B. Gut-associated microbes of Drosophila melanogaster. Gut Microbes 2012; 3.

18. Obadia B, Güvener ZT, Zhang V, Ceja-Navarro JA, Brodie EL, Ja WW, et al. Probabilistic Invasion Underlies Natural Gut Microbiome Stability. Curr Biol 2017; 27: 1999-2006.e8.

19. Koyle ML, Veloz M, Judd AM, Wong ACN, Newell PD, Douglas AE, et al. Rearing the Fruit Fly Drosophila melanogaster Under Axenic and Gnotobiotic Conditions. 2016; e54219.

20. Newell PD, Douglas AE. Interspecies Interactions Determine the Impact of the Gut Microbiota on Nutrient Allocation in Drosophila melanogaster. Appl Environ Microbiol 2013; 80: 788-796.

21. Walters AW, Matthews MK, Hughes RC, Malcolm J, Rudman S, Newell PD, et al. The microbiota influences the Drosophila melanogaster life history strategy. bioRxiv . 2018.

22. Chaston JM, Dobson AJ, Newell PD, Douglas AE. Host genetic control of the microbiota mediates Drosophila nutritional phenotype. Appl Environ Microbiol 2015; 82.

23. Dobson AJ, Chaston JM, Newell PD, Donahue L, Hermann SL, Sannino DR, et al. Corrigendum: Host genetic determinants of microbiota-dependent nutrition revealed by genome-wide analysis of Drosophila melanogaster. Nat Commun 2015; 6: 7296.

24. Gould AL, Zhang V, Lamberti L, Jones EW, Obadia B, Korasidis N, et al. Microbiome interactions shape host fitness. Proc Natl Acad Sci U S A 2018; 115: E11951E11960.

25. Rudman SM, Greenblum S, Hughes RC, Rajpurohit S, Kiratli O, Lowder DB, et al. Microbiome composition shapes rapid genomic adaptation of Drosophila melanogaster. bioRxiv . 2019. , 632257

26. Bertness MD, Callaway R. Positive interactions in communities. Trends Ecol Evol 1994; 9: 191-193.

27. Bronstein JL. Conditional outcomes in mutualistic interactions. Trends Ecol Evol 1994; 9: 214-217.

28. David AS, Thapa-Magar KB, Afkhami ME. Microbial mitigation-exacerbation continuum: a novel framework for microbiome effects on hosts in the face of stress. Ecology 2018; 99: 517-523.

29. Martino ME, Joncour P, Leenay R, Gervais H, Shah M, Hughes S, et al. Bacterial Adaptation to the Host's Diet Is a Key Evolutionary Force Shaping DrosophilaLactobacillus Symbiosis. Cell Host Microbe 2018; 24: 109-119.e6.

30. Erkosar B, Yashiro E, Zajitschek F, Friberg U, Maklakov AA, van der Meer JR, et al. Host diet mediates a negative relationship between abundance and diversity of Drosophila gut microbiota. Ecol Evol 2018; 8: 94919502.

31. Erkosar B, Kolly S, van der Meer JR, Kawecki TJ. Adaptation to Chronic Nutritional Stress Leads to 
Reduced Dependence on Microbiota in Drosophila melanogaster. MBio 2017; 8 .

32. Musselman LP, Fink JL, Narzinski K, Ramachandran PV, Hathiramani SS, Cagan RL, et al. A high-sugar diet produces obesity and insulin resistance in wild-type Drosophila. Dis Model Mech 2011; 4: 842-849.

33. Na J, Musselman LP, Pendse J, Baranski TJ, Bodmer R, Ocorr K, et al. A Drosophila model of high sugar dietinduced cardiomyopathy. PLoS Genet 2013; 9: e1003175.

34. Rovenko BM, Kubrak OI, Gospodaryov DV, Perkhulyn NV, Yurkevych IS, Sanz A, et al. High sucrose consumption promotes obesity whereas its low consumption induces oxidative stress in Drosophila melanogaster. J Insect Physiol 2015; 79: 42-54.

35. Musselman LP, Fink JL, Ramachandran PV, Patterson BW, Okunade AL, Maier E, et al. Role of fat body lipogenesis in protection against the effects of caloric overload in Drosophila. J Biol Chem 2013; 288: 80288042.

36. Huang J-H, Douglas AE. Consumption of dietary sugar by gut bacteria determines Drosophila lipid content. Biol Lett 2015; 11: 20150469.

37. Grenier JK, Roman Arguello J, Moreira MC, Gottipati S, Mohammed J, Hackett SR, et al. Global Diversity LinesA Five-Continent Reference Panel of Sequenced Drosophila melanogaster Strains. G3: Genes, Genomes, Genetics 2015; 5: 593-603.

38. Mandal S, Van Treuren W, White RA, Eggesbø M, Knight R, Peddada SD. Analysis of composition of microbiomes: a novel method for studying microbial composition. Microb Ecol Health Dis 2015; 26: 27663.

39. Winans NJ, Walter A, Chouaia B, Chaston JM, Douglas AE, Newell PD. A genomic investigation of ecological differentiation between free-living and Drosophilaassociated bacteria. Mol Ecol 2017.

40. Therneau TM, Lumley T. Package 'survival'. $R$ Top Doc 2015; 128: 112.

41. Kassambara A. Kosinski M. survminer: Drawing survival curves using 'ggplot2,' 2018. URL https://CRAN $R$-project org/package $=$ survminer $R$ package version 0 4 2018; 3.

42. Magnusson A, Skaug H, Nielsen A, Berg C, Kristensen $\mathrm{K}$, Maechler M, et al. glmmTMB: generalized linear mixed models using template model builder. $R$ package version 01 2017; 3 .

43. Fox J, Weisberg S, Adler D, Bates D, Baud-Bovy G, Ellison S, et al. Package 'car'. Vienna: R Foundation for Statistical Computing 2012.

44. Lenth R. emmeans: Estimated marginal means, aka leastsquares means. R package version 1.4. 3.01. 2019.

45. Tennessen JM, Barry WE, Cox J, Thummel CS. Methods for studying metabolism in Drosophila. Methods 2014; 68: $105-115$.

46. Bojanova DP, Bordenstein SR. Fecal Transplants: What Is Being Transferred? PLoS Biol 2016; 14: e1002503.
47. Gilbert JA, Lynch SV. Community ecology as a framework for human microbiome research. Nat Med 2019; 25: 884-889.

48. Duvallet C, Zellmer C, Panchal P, Budree S, Osman M, Alm EJ. Framework for rational donor selection in fecal microbiota transplant clinical trials. PLoS One 2019; 14: $\mathrm{e} 0222881$.

49. Chandler JA, Lang JM, Bhatnagar S, Eisen JA, Kopp A. Bacterial communities of diverse Drosophila species: ecological context of a host-microbe model system. PLoS Genet 2011; 7: e1002272.

50. Staubach F, Baines JF, Künzel S, Bik EM, Petrov DA. Host species and environmental effects on bacterial communities associated with Drosophila in the laboratory and in the natural environment. PLoS One 2013; 8: e70749.

51. Adair KL, Wilson M, Bost A, Douglas AE. Microbial community assembly in wild populations of the fruit fly Drosophila melanogaster. ISME J 2018; 12: 959-972.

52. Wang Y, Kapun M, Waidele L, Kuenzel S, Bergland AO, Staubach F. Common structuring principles of the Drosophila melanogaster microbiome on a continental scale and between host and substrate. Environ Microbiol Rep 2020; 12: 220-228.

53. Kersters K, Lisdiyanti P, Komagata K, Swings J. The Family Acetobacteraceae: The Genera Acetobacter, Acidomonas, Asaia, Gluconacetobacter, Gluconobacter, and Kozakia. In: Dworkin M, Falkow S, Rosenberg E, Schleifer K-H, Stackebrandt E (eds). The Prokaryotes: Volume 5: Proteobacteria: Alpha and Beta Subclasses. 2006. Springer New York, New York, NY, pp 163-200.

54. Shin SC, Kim S-H, You H, Kim B, Kim AC, Lee K-A, et al. Drosophila microbiome modulates host developmental and metabolic homeostasis via insulin signaling. Science 2011; 334: 670-674.

55. Chaston JM, Newell PD, Douglas AE. Metagenomewide association of microbial determinants of host phenotype in Drosophila melanogaster. MBio 2014; 5: e01631-14.

56. Yasuda K, Oh K, Ren B, Tickle TL, Franzosa EA, Wachtman LM, et al. Biogeography of the intestinal mucosal and lumenal microbiome in the rhesus macaque. Cell Host Microbe 2015; 17: 385-391.

57. Ingala MR, Simmons NB, Wultsch C, Krampis K, Speer KA, Perkins SL. Comparing Microbiome Sampling Methods in a Wild Mammal: Fecal and Intestinal Samples Record Different Signals of Host Ecology, Evolution. Front Microbiol 2018; 9: 803.

58. Paramsothy S, Paramsothy R, Rubin DT, Kamm MA, Kaakoush NO, Mitchell HM, et al. Faecal Microbiota Transplantation for Inflammatory Bowel Disease: A Systematic Review and Meta-analysis. J Crohns Colitis 2017; 11: 1180-1199.

59. Martínez-Mota R, Kohl KD, Orr TJ, Denise Dearing M. Natural diets promote retention of the native gut microbiota in captive rodents. ISME J 2020; 14: 67-78. 
60. Flatt T. Life-History Evolution and the Genetics of Fitness Components in Drosophila melanogaster. Genetics 2020; 214: 3-48.

61. Grimaldi D, Jaenike J. Competition in Natural Populations of Mycophagous Drosophila. Ecology 1984; 65: 1113-1120.

62. Nunney L. Drosophila on Oranges: Colonization, Competition, and Coexistence. Ecology 1990; 71: 1904 1915.

63. Walters AW, Hughes RC, Call TB, Walker CJ, Wilcox $\mathrm{H}$, Petersen SC, et al. The microbiota influences the Drosophila melanogaster life history strategy. Mol Ecol 2019.

64. Smith P, Willemsen D, Popkes M, Metge F, Gandiwa E, Reichard M, et al. Regulation of life span by the gut microbiota in the short-lived African turquoise killifish. Elife 2017; 6.

65. Schwab DB, Riggs HE, Newton ILG, Moczek AP. Developmental and Ecological Benefits of the Maternally Transmitted Microbiota in a Dung Beetle. $\mathrm{Am}$ Nat 2016; 188: 679-692.

66. Panke-Buisse K, Poole AC, Goodrich JK, Ley RE, KaoKniffin J. Selection on soil microbiomes reveals reproducible impacts on plant function. ISME J 2015; 9: 980-989.

67. Metcalf CJE, Henry LP, Rebolleda-Gómez M, Koskella B. Why Evolve Reliance on the Microbiome for Timing of Ontogeny? MBio 2019; 10.

68. Storelli G, Defaye A, Erkosar B, Hols P, Royet J, Leulier F. Lactobacillus plantarum promotes Drosophila systemic growth by modulating hormonal signals through TOR-dependent nutrient sensing. Cell Metab 2011; 14: 403-414.

69. Buchon N, Broderick NA, Lemaitre B. Gut homeostasis in a microbial world: insights from Drosophila melanogaster. Nat Rev Microbiol 2013; 11: 615-626.

70. Alberdi A, Aizpurua O, Bohmann K, Zepeda-Mendoza ML, Gilbert MTP. Do vertebrate gut metagenomes confer rapid ecological adaptation? Trends Ecol Evol 2016; 31: 689-699.

71. Carrier TJ, Reitzel AM. The Hologenome Across Environments and the Implications of a Host-Associated Microbial Repertoire. Front Microbiol 2017; 8: 802.

72. Henry LP, Bruijning M, Forsberg SKG, Ayroles JF. Can the microbiome influence host evolutionary trajectories? bioRxiv 2019; 700237.

73. David AS, Thapa-Magar KB, Menges ES, Searcy CA, Afkhami ME. Do plant-microbe interactions support the Stress Gradient Hypothesis? Ecology 2020; e03081.

74. Petipas RH, Wruck AC, Geber MA. Microbe-mediated local adaptation to limestone barrens is context dependent. Ecology 2020; 84: 93.

75. Mueller EA, Wisnoski NI, Peralta AL, Lennon JT. Microbial rescue effects: How microbiomes can save hosts from extinction. Funct Ecol 2020; 33.

76. Ridley EV, Wong ACN, Douglas AE. Microbe-
Dependent and Nonspecific Effects of Procedures To Eliminate the Resident Microbiota from Drosophila melanogaster. Appl Environ Microbiol 2013; 79: 3209 3214.

77. Heys C, Lizé A, Blow F, White L, Darby A, Lewis ZJ. The effect of gut microbiota elimination in Drosophila melanogaster: a how-to guide for host--microbiota studies. Ecol Evol 2018; 8: 4150-4161.

78. Long A, Liti G, Luptak A, Tenaillon O. Elucidating the molecular architecture of adaptation via evolve and resequence experiments. Nat Rev Genet 2015; 16: $567-$ 582.

79. Schlötterer C, Kofler R, Versace E, Tobler R, Franssen SU. Combining experimental evolution with nextgeneration sequencing: a powerful tool to study adaptation from standing genetic variation. Heredity 2015; 114: 431-440.

80. Henry LP, Ayroles JF. The microbiome responds to Evolve and Resequence experiments in Drosophila melanogaster. bioRxiv 2020; 2020.03.19.999078.

81. Hoang KL, Morran LT, Gerardo NM. Experimental Evolution as an Underutilized Tool for Studying Beneficial Animal-Microbe Interactions. Front Microbiol 2016; 07. 


\section{TABLES}

Table 1: ANCOM results for differentially abundant ASVs from C and HS fly microbiomes. Taxonomy was characterized to the lowest level an assignment could be made, and most ASVs were classified to species level.

\begin{tabular}{|c|c|c|c|c|}
\hline ASV ID & $\mathrm{clr}$ & W & sig & taxonomy \\
\hline d84f3e1a7dbb97bc4695b954c8a2d2b2 & 4.30129864 & 25 & HS fly & Acetobacteraceae \\
\hline fb59adcbdd838dc7853f7388afb62b5a & 7.82830283 & 25 & HS fly & Acetobacter pasteurianus \\
\hline 9329896cd08f88a88296d921369cd8ea & -3.4609509 & 20 & C fly & Algoriella xinjiangensis \\
\hline f0d11f9f8ba452d5485a79e0b31b538f & -3.6539534 & 20 & C fly & Acetobacter indonesiensis \\
\hline 3f2be3d57ac38f60b0b9c56fa5857f63 & -2.7057494 & 18 & Neutral & Algoriella xinjiangensis \\
\hline 70e118808cdc81b5b2670837c7833d8d & -3.3688051 & 18 & Neutral & Acetobacter persici \\
\hline dd4826376c1de49b8687c4f882d3bcf3 & -2.1106948 & 17 & Neutral & Enterobacteriaceae \\
\hline $606 a a 8 c 7117 f 801 d c 5 c c c 8 c 2 a 78 c 68 c 3$ & -1.7000214 & 16 & Neutral & Commensalibacter \\
\hline 1c735b8a08dcbd5ca158987733234114 & -1.6719837 & 15 & Neutral & Algoriella xinjiangensis \\
\hline da74ac9885f0aa2d29ec4338b46aa051 & 2.00310762 & 13 & Neutral & Orbaceae \\
\hline 4043baca9447b9860efabab56603efc0 & 0.79635173 & 10 & Neutral & Acetobacter \\
\hline 61fd19eda2dee9c93613432491556a99 & 0.69392446 & 10 & Neutral & Alphaproteobacteria \\
\hline 7070daee4850cf801719eab99ce88a82 & 0.96974134 & 10 & Neutral & Commensalibacter \\
\hline b3ccbf1d4fc85411f948429591f371cf & 0.59968784 & 10 & Neutral & Pseudomonas \\
\hline ca7279bfdcb977287193c567f96b025c & 0.75112887 & 10 & Neutral & Acetobacter indonesiensis \\
\hline 0a04cded1e993516d5cdc851bfe07180 & -1.6048832 & 9 & Neutral & Algoriella xinjiangensis \\
\hline 6e4961fe480153e97ebdcf8fca4913fb & 0.99515931 & 9 & Neutral & Providencia \\
\hline 8dc187c9810636b7199c030caaaf0694 & 0.58697562 & 9 & Neutral & Morganella \\
\hline 9f9bb5e1a63c5a0820d1571839534a2f & 0.62165362 & 9 & Neutral & Commensalibacter \\
\hline 677d95c5a0bc1c25435861fe262cb487 & 0.50699766 & 8 & Neutral & Alphaproteobacteria \\
\hline 4af26492bbe6d0d4980bc82c2f08f32d & 0.3940931 & 7 & Neutral & Providencia \\
\hline ad8b60b9428bc76f3b50e428f4f5a29f & 0.41862229 & 7 & Neutral & Stenotrophomonas \\
\hline f72576c61dde7b9b6ad683dea71b105f & 0.06411428 & 7 & Neutral & Enterobacteriaceae \\
\hline $3 c 3 c 65 e 801 e 07 d 1 b e 83 b f a 6 d 962 b 5627$ & -0.3759816 & 6 & Neutral & Commensalibacter \\
\hline 681de033774ada405895dd81abc7593f & -0.5750105 & 6 & Neutral & Providencia rettgeri \\
\hline 97b76e52dafa224603888fb56ef0b831 & -0.3031253 & 5 & Neutral & Commensalibacter \\
\hline
\end{tabular}


Table 2A: Analysis of deviance for Cox model with interactions between fly genotype, microbiome, and diet for developmental timing in the Acetobacter transplant.

\begin{tabular}{|c|c|c|c|c|c|}
\hline Category & Log-likelihood & $x^{2}$ & Df & & $P$ value \\
\hline Null & -60640 & & & & \\
\hline Fly genotype & -60584 & 111.642 & & 1 & $<2.20 \mathrm{E}-16^{* * *}$ \\
\hline Acetobacter & -60517 & 134.874 & & 2 & $<2.20 \mathrm{E}-16^{* * *}$ \\
\hline Diet & -58732 & 3568.585 & & 1 & $<2.20 \mathrm{E}-16^{\star * *}$ \\
\hline Fly x Acetobacter & -58675 & 115.244 & & 2 & $<2.20 \mathrm{E}-16^{* * *}$ \\
\hline Fly x Diet & -58658 & 34.288 & & 1 & 4.75E-09*** \\
\hline Acetobacter $x$ Diet & -58523 & 268.613 & & 2 & $<2.20 \mathrm{E}-16^{* * *}$ \\
\hline Fly x Acetobacter x Diet & -58500 & 47.625 & & 2 & $4.55 \mathrm{E}-11^{* * *}$ \\
\hline
\end{tabular}

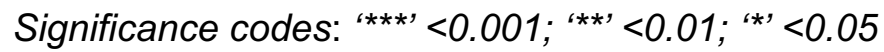

Table 2B: Model selection statistics for developmental timing in the Acetobacter transplant. Model 1 is without interactions, while Model 2 includes all two and three-way interactions.

\begin{tabular}{|c|c|c|c|c|c|}
\hline Model & Log-likelihood & $x^{2}$ & $\mathrm{X}^{2} \mathrm{Df}$ & & $P(>\mid$ Chi $\mid)$ \\
\hline Model 1 & -58732 & & & & \\
\hline Model 2 & -58500 & 465.77 & & 7 & $<2.20 \mathrm{E}-16^{\star * *}$ \\
\hline
\end{tabular}

Table 3A: Fixed effects for fecundity in the Acetobacter transplant. Significance was evaluated using Type III Wald $\mathrm{F}$ tests with Kenward-Roger degrees of freedom.

\begin{tabular}{|c|c|c|c|}
\hline Category & $x^{2}$ & $\mathrm{Df}$ & $P$ value \\
\hline Intercept & 637.8446 & 1 & $<2.20 \mathrm{E}-16^{\star * *}$ \\
\hline Fly genotype & 4.0333 & 1 & $0.0446106^{*}$ \\
\hline Acetobacter & 39.7839 & 2 & $2.30 \mathrm{E}-09^{* * *}$ \\
\hline Diet & 53.9716 & 1 & $2.03 \mathrm{E}-13^{* \star *}$ \\
\hline Fly $x$ Acetobacter & 7.787 & 2 & $0.0203736^{*}$ \\
\hline Fly $x$ Diet & 8.4003 & 1 & $0.0037517^{* *}$ \\
\hline Acetobacter $x$ Diet & 14.4492 & 2 & $0.0007285^{\star * *}$ \\
\hline Fly $\times$ Acetobacter $\times$ Diet & 16.3387 & 2 & $0.0002832^{* * *}$ \\
\hline
\end{tabular}

Table 3B: Model selection statistics for fecundity in the Acetobacter transplant. Model 1 is without interactions, while Model 2 includes all two and three-way interactions.

\begin{tabular}{|c|c|c|c|c|c|c|c|c|}
\hline Model & $\mathrm{Df}$ & AIC & $\mathrm{BIC}$ & logLik & deviance & $x^{2}$ & $X^{2} D f$ & $P$ value \\
\hline Model 1 & 7 & 6333.5 & 6365 & -3159.8 & 6319.5 & & & \\
\hline Model 2 & 14 & $\begin{array}{l}6296.8 \\
\text { Signifiti }\end{array}$ & 6359.8 & $\begin{array}{l}-3134.4 \\
*_{* * * *}<<00\end{array}$ & 6268.8 & $\begin{array}{l}50.753 \\
x^{*}<0.05\end{array}$ & 7 & $1.03 \mathrm{E}-08^{* * *}$ \\
\hline
\end{tabular}


Table 4A: Fixed effects for protein (as a proxy for size) in the Acetobacter transplant. Significance was evaluated using Type III Wald F tests with Kenward-Roger degrees of freedom.

\begin{tabular}{lrrrl}
\hline Category & F & Df & Df.res & $P$ value \\
\hline Intercept & 229.8272 & 1 & 7.004 & $1.30 \mathrm{E}-06^{* * *}$ \\
Fly genotype & 21.9301 & 1 & 78.06 & $1.17 \mathrm{E}-05^{* * *}$ \\
Diet & 11.3077 & 1 & 78.062 & $0.001198^{* *}$ \\
Acetobacter & 0.9362 & 2 & 78.013 & 0.396492 \\
Fly x Diet & 18.3091 & 1 & 78.068 & $5.28 \mathrm{E}-05^{\star * *}$ \\
Fly x Acetobacter & 0.3589 & 2 & 78.066 & 0.699579 \\
Diet x Acetobacter & 3.2618 & 2 & 78.156 & $0.043595^{*}$
\end{tabular}

Significance codes: “***'<0.001; ‘**' $<0.01$; ‘*’ $<0.05$

Table 4B: Model selection statistics for protein in the Acetobacter transplant. Model 1 is without interactions, while Model 2 includes all two-way interactions. The three-way interaction was not significant and removed from the analysis.

\begin{tabular}{|c|c|c|c|c|c|c|c|c|}
\hline Model & Df & $\mathrm{AIC}$ & $\mathrm{BIC}$ & $\log \mathrm{Lik}$ & deviance & $x^{2}$ & $X^{2} D f$ & $P$ value \\
\hline Model 1 & 7 & -149.89 & -132.01 & 81.943 & -163.89 & & & \\
\hline Model 2 & 12 & -164.99 & -134.34 & 94.494 & -188.99 & 25.102 & 5 & $0.0001332^{\star * *}$ \\
\hline
\end{tabular}

Table 5A: Fixed effects for triglyceride accumulation in the Acetobacter transplant. Significance was evaluated using Type III Wald F tests with Kenward-Roger degrees of freedom.

\begin{tabular}{|c|c|c|c|c|}
\hline Category & $\mathrm{F}$ & Df & Df.res & $P$ value \\
\hline Intercept & 30.7704 & 1 & 7.002 & $0.0008616^{* * *}$ \\
\hline Fly genotype & 12.0201 & 1 & 78.037 & $0.0008591^{* * *}$ \\
\hline Diet & 27.3194 & 1 & 78.039 & 1.40E-06 $06^{* * *}$ \\
\hline Acetobacter & 0.028 & 2 & 78.008 & 0.9724439 \\
\hline Fly x Diet & 8.9996 & 1 & 78.043 & $0.0036234^{* *}$ \\
\hline Fly $\times$ Acetobacter & 1.7817 & 2 & 78.041 & 0.1751283 \\
\hline Diet x Acetobacter & 1.771 & 2 & 78.098 & 0.1769287 \\
\hline
\end{tabular}

Table 5B: Model selection statistics for triglyceride in the Acetobacter transplant. Model 1 is without interactions, while Model 2 includes all two-way interactions. The three-way interaction was not significant and removed from the analysis.

\begin{tabular}{|c|c|c|c|c|c|c|c|c|}
\hline Model & Df & AIC & $\mathrm{BIC}$ & logLik & deviance & $x^{2}$ & $\mathrm{X}^{2} \mathrm{Df}$ & $P$ value \\
\hline Model 1 & 7 & 440.31 & 458.19 & -213.16 & 426.31 & & & \\
\hline Model 2 & 12 & 433.09 & 463.73 & -204.54 & 409.09 & 17.226 & & $0.00409^{* *}$ \\
\hline
\end{tabular}


bioRxiv preprint doi: https://doi.org/10.1101/2020.09.21.306779; this version posted September 22, 2020. The copyright holder for this preprint (which was not certified by peer review) is the author/funder. All rights reserved. No reuse allowed without permission.

Henry et al., 09-17-2020 bioRxiv 


\section{SUPPLEMENTARY FIGURES}

\section{Supp. Fig. 1: SUPPLEMENTARY FIGURES}

A

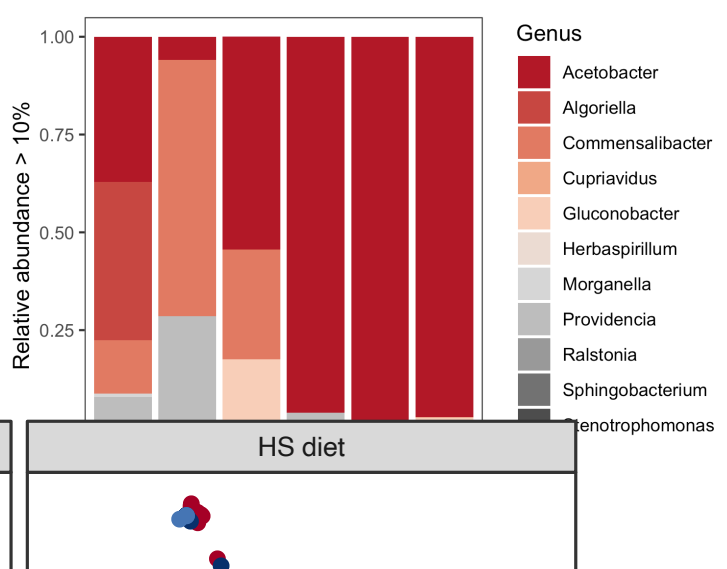

C

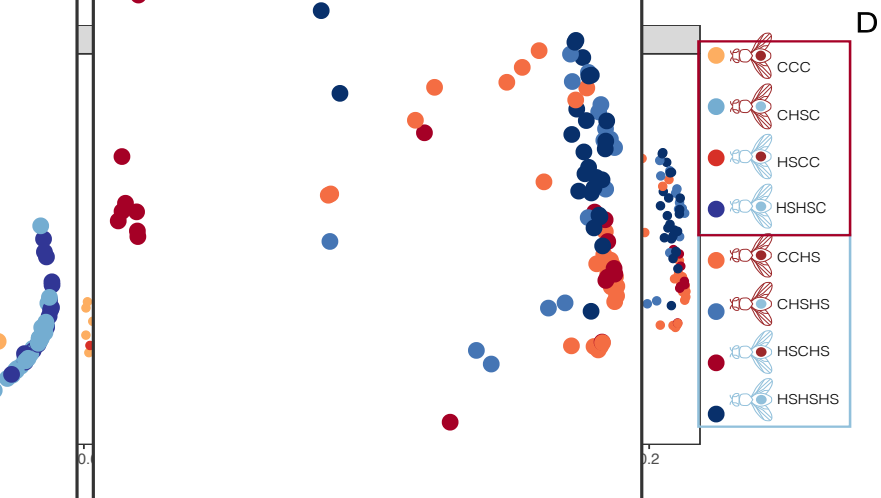

B
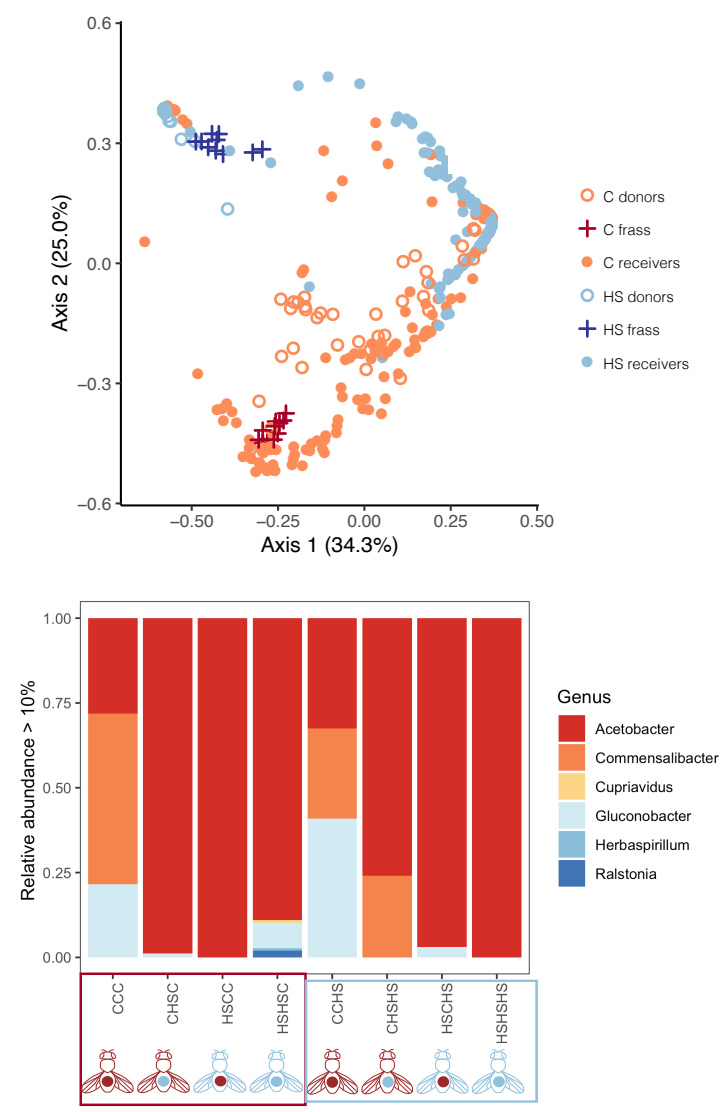

Supp. Fig. 1: 16S rRNA characterization of microbiome transplant.

A) Relative abundance at the genus level for donors, frass, and recipients in microbiome transplant. Each step of the transplant (i.e., donor, frass, recipient) shows overlap in only a few species. B) PCoA plots for Bray-Curtis distance based on relative abundance of ASVs for the microbiome transplant. Points represent individual flies, except for the frass (which was pooled from 250 flies and 6-8 replicates sequenced). Axis 1 mostly separates between frass and flies, while Axis 2 shows some separation between C and HS microbiomes. C) PCoA plots for Bray-Curtis distance for only the recipients, separated by diet. On the $C$ diet, flies that received the $C$ microbiome were distinct from those with the HS microbiome, suggesting fidelity of the microbiome transplant. However, on the HS diet, the microbiome transplant did not reconstitute differences between C and HS microbiomes in the recipient. D) Relative abundance of genera for each treatment in the recipients. The $C$ recipients were composed of primarily Acetobacter and Commensalibacter genera, while only Acetobacter genera in HS recipients, especially on HS diets. 
A

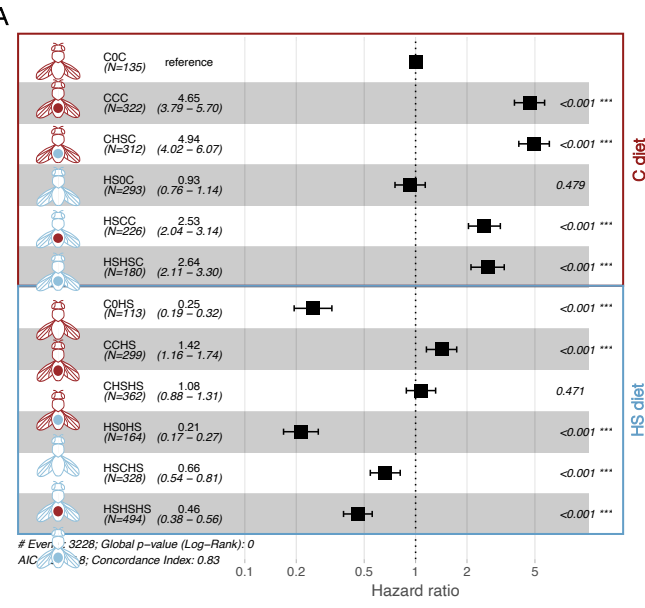

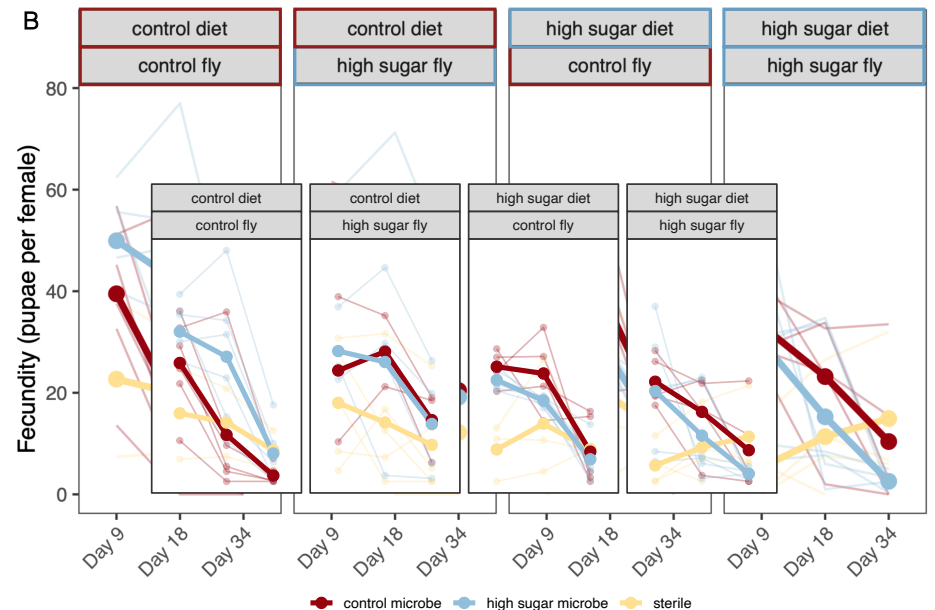

Supp. Fig. 2: Life history effects of the microbiome transplant.

A) Hazard ratio plot for microbiome effects on developmental time. Boxplots show hazard ratios and $95 \%$ confidence intervals for each treatment relative to COC. On the C diet, both fly genotypes with either C or HS microbiomes developed faster than sterile flies. However, on $\mathrm{HS}$ diet, and the $\mathrm{C}$ microbiome accelerated while HS microbiome slowed development. The interaction effects on the Cox proportional hazards model best explained variation in development (log-likelihood $=-21807, d f=7, X^{2}=78.026, p<0.001$ ), suggesting that fly evolution and environment shape phenotypic effects of the microbiome. B) Fecundity effects of the microbiome transplant at three timepoints over 34 days. Plots are faceted by fly genotype and diet. Thick lines with dots represent the average fecundity for the microbiome treatment. Thinner lines represent the replicates. Fecundity declined over time $\left(F_{2,105.3}=49.4, p<0.0001\right)$ and was shaped by both $\operatorname{diet}\left(F_{2,49}=4.35\right.$, $\mathrm{p}=0.02)$ and microbiome $\left(\mathrm{F}_{1,50}=5.41, \mathrm{p}=0.02\right)$. 
Henry et al., 09-17-2020 bioRxiv

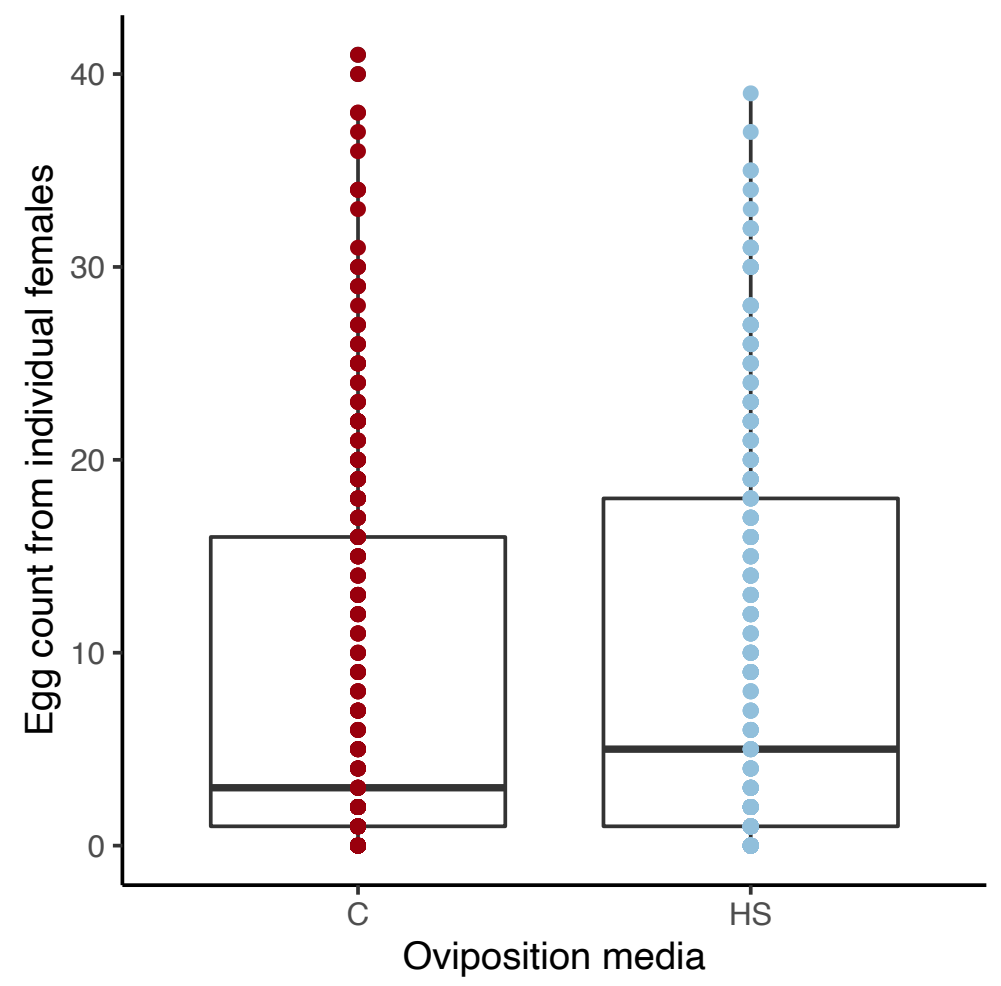

Supp. Fig. 3: Oviposition media differences.

No difference for egg counts between C and HS oviposition media (Kruskall-Wallis $X^{2}=2.85, d f=1, p=0.09$ ). 

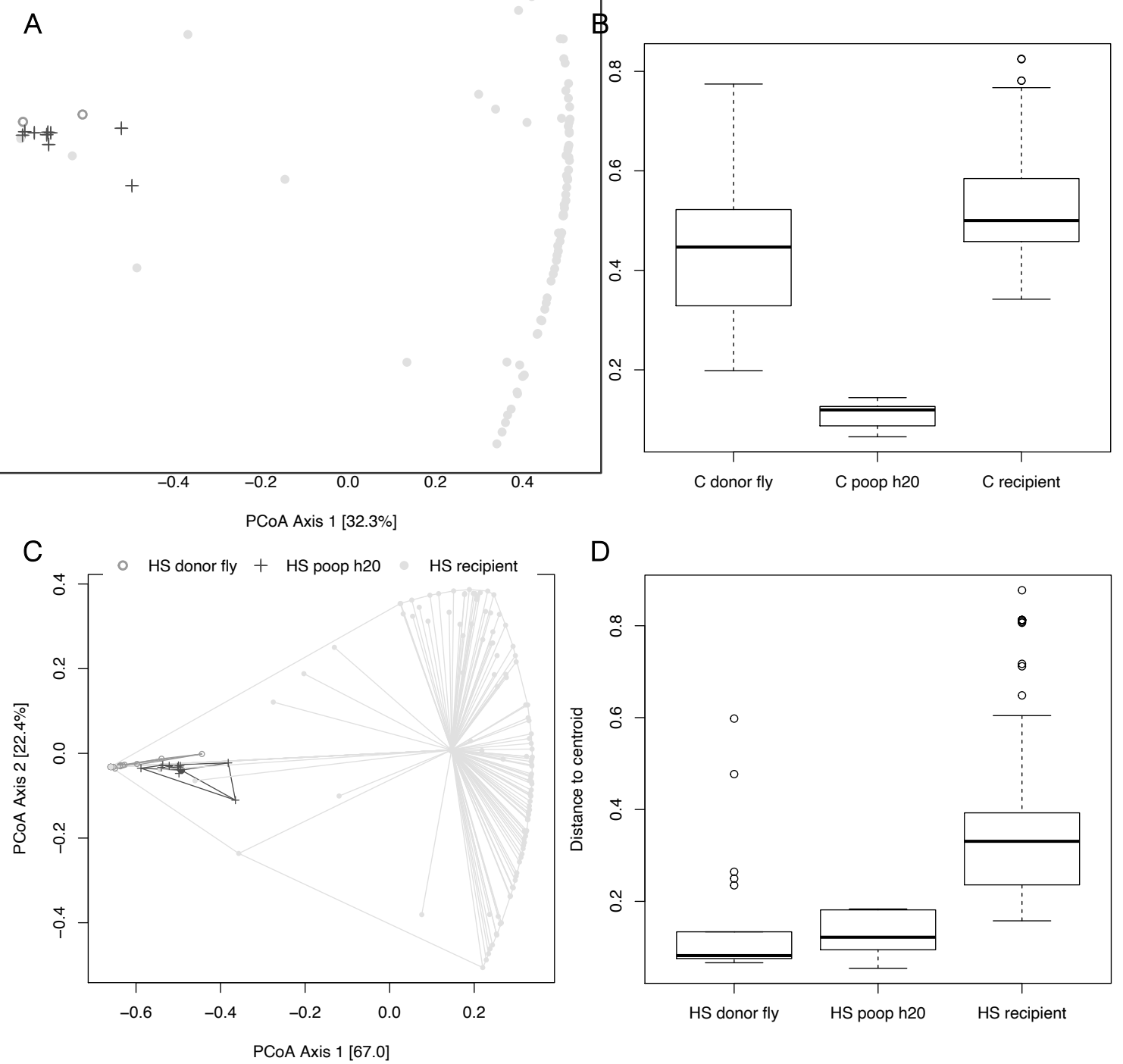

Supp. Fig 4: Differences in betadispersion between donor and recipients in microbiome transplant.

A) The relationship between donor, frass, and recipient on the $C$ diet based on Bray-Curtis dissimilarity. Each point represents a fly, while the lines show the distance to mean value for each. B) Boxplot for centroid dispersion on the $\mathrm{C}$ diet. C) The relationship between donor, frass, and recipient on the HS diet based on BrayCurtis dissimilarity. D) Boxplot for centroid dispersion on the HS diet. 


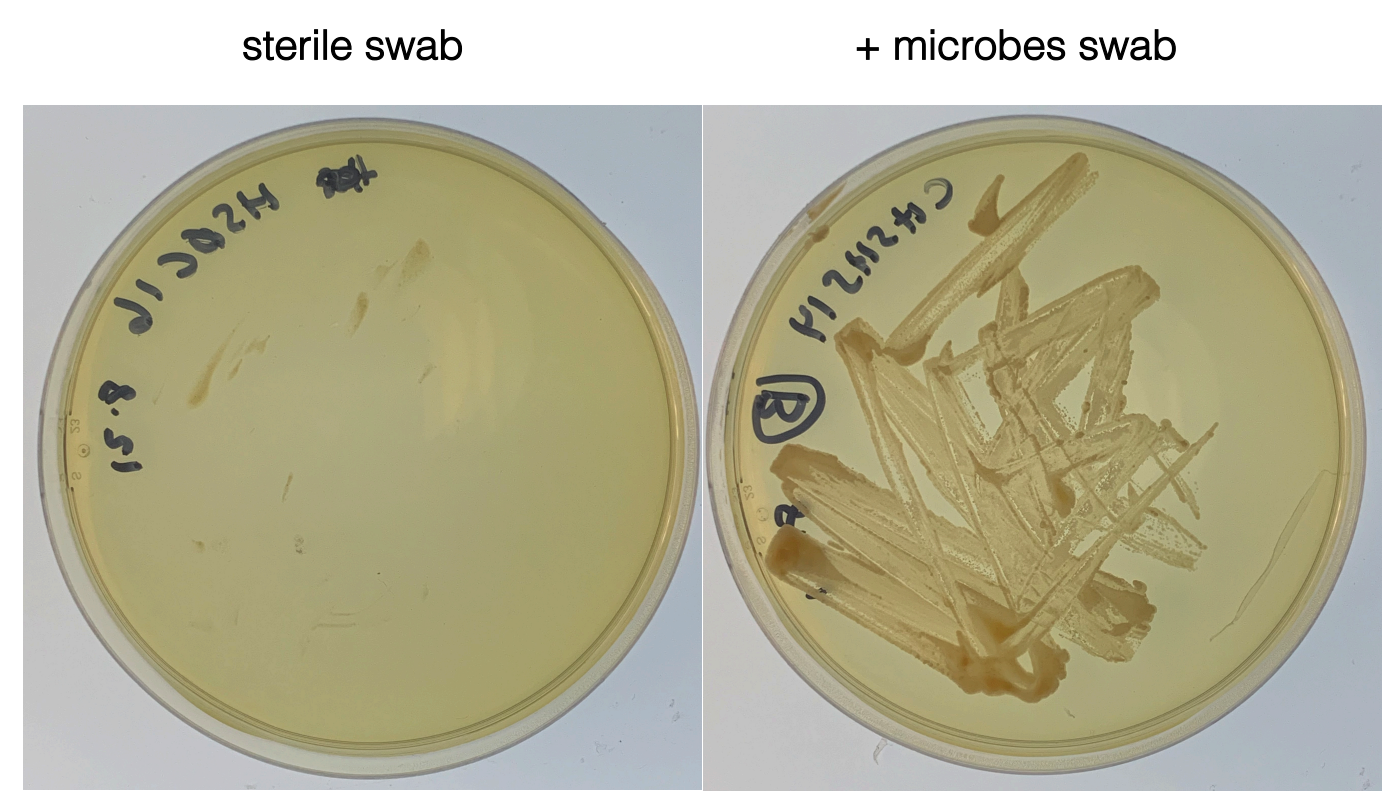

\section{Supp. Fig. 5: Representative images for sterility checks during Acetobacter transplant.}

Food was swabbed during development and streaked on MRS plates. Sterile swab shows no growth and only food smudged. Microbes swab shows microbial growth. 


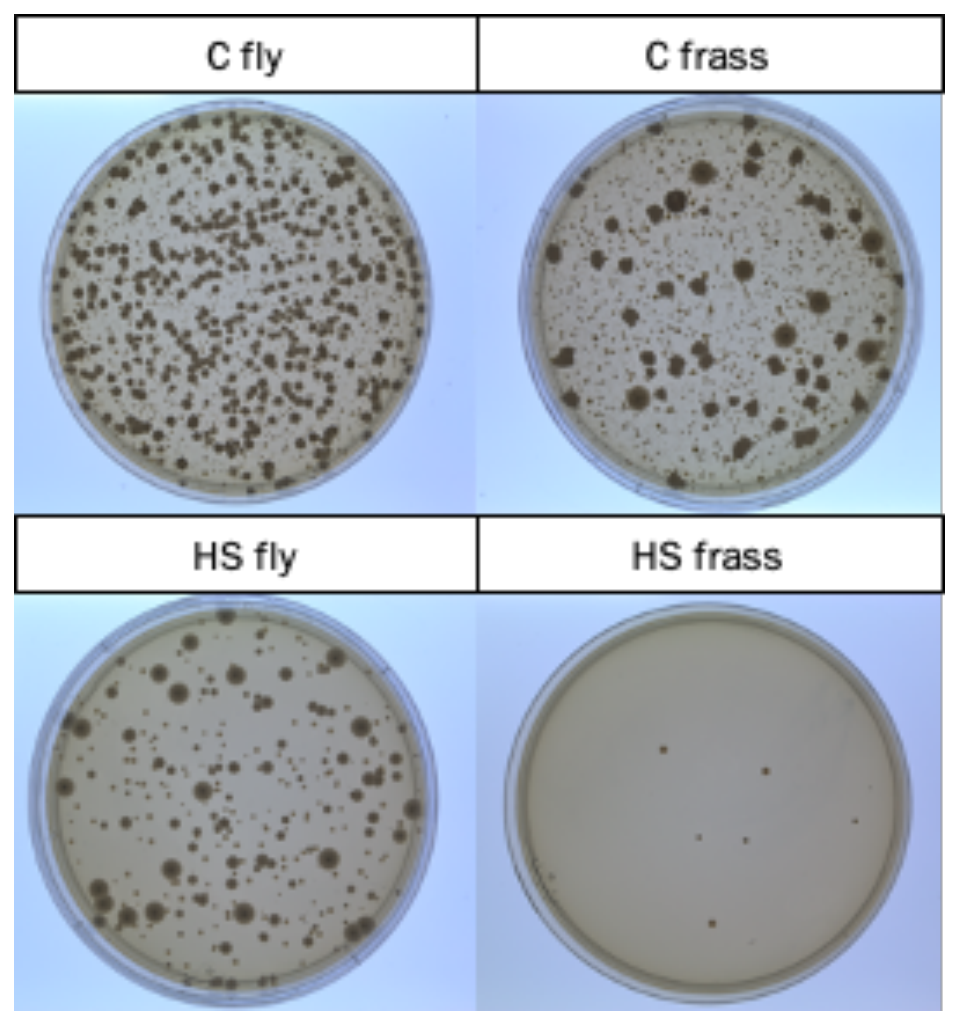

Supp. Fig. 6: Differences in bacterial abundance between flies and frass for the $\mathrm{C}$ and HS fly genotypes. Figures show cultured bacteria ( $50 \mu \mathrm{l}$ of $500 \mu \mathrm{l}$ homogenate of 5 female flies or $50 \mu$ l of frass from 200 flies) on MRS media incubated for 3 days. $\mathrm{C}$ flies harbor higher amounts of bacteria than HS flies. Bacteria in HS frass is especially low abundance. 


\section{SUPPLEMENTARY TABLES}

Table 6: Comparison of centroid dispersion for donors, frass, and recipients in the microbiome transplant with 999 permutations.

\begin{tabular}{|c|c|c|c|c|c|}
\hline Category & Df & SS & MS & $\mathrm{F}$ & $P$ value \\
\hline Microbiome source & 5 & 5.715 & 1.143 & 36.282 & 0.001 \\
\hline Residuals & 334 & 10.522 & 0.0315 & & \\
\hline
\end{tabular}

Table 7: Comparison of microbial community composition for recipients on C diets using PERMANOVA with 999 permutations.

\begin{tabular}{|c|c|c|c|c|c|c|}
\hline Category & $\mathrm{Df}$ & SS & MS & $\mathrm{F}$ & $\mathrm{R}^{2}$ & $P$ value \\
\hline Fly genotype & 1 & 0.6954 & 0.6954 & 6.129 & 0.02344 & 0.004 \\
\hline Microbiome & 1 & 12.6455 & 12.6455 & 111.45 & 0.42627 & 0.001 \\
\hline Fly genotype $x$ Microbiome & 1 & 0.4395 & 0.4395 & 3.874 & 0.01482 & 0.01 \\
\hline Residuals & 140 & 15.8849 & 0.1135 & 0.5354 & & \\
\hline Total & 143 & 29.6653 & 1 & & & \\
\hline
\end{tabular}

Table 8: Comparison of microbial community composition for recipients on HS diets using PERMANOVA with 999 permutations.

\begin{tabular}{lrrrrrr}
\hline Category & Df & \multicolumn{1}{l}{ SS } & MS & \multicolumn{2}{l}{$\mathrm{R}^{2}$} & \multicolumn{1}{l}{$P$ value } \\
\hline Fly genotype & 1 & 1.8347 & 1.83472 & 8.5998 & 0.06509 & 0.001 \\
Microbiome & 1 & 1.1485 & 1.14846 & 5.3831 & 0.04074 & 0.006 \\
Fly genotype x Microbiome & 1 & 1.3113 & 1.3113 & 6.1464 & 0.04652 & 0.003 \\
Residuals & 112 & 23.8945 & 0.21334 & 0.84765 & & \\
Total & 115 & 28.189 & 1 & & &
\end{tabular}

Table 9: Comparisons of microbial community composition for all recipients using PERMANOVA with 999 permutations.

\begin{tabular}{lrrrrrr}
\hline Category & Df & \multicolumn{1}{c}{ SS } & \multicolumn{1}{c}{ MS } & \multicolumn{1}{l}{ 年 } & \multicolumn{2}{l}{$P$ value } \\
\hline Fly genotype & 1 & 1.189 & 1.1891 & 22.026 & 0.0172 & 0.001 \\
Microbiome & 1 & 9.302 & 9.3019 & 172.301 & 0.13455 & 0.001 \\
Diet & 1 & 10.577 & 10.5766 & 195.913 & 0.15299 & 0.001 \\
Fly genotype x Microbiome & 1 & 0.339 & 0.3387 & 6.274 & 0.0049 & 0.001 \\
Fly genotype x Diet & 1 & 1.656 & 1.6562 & 30.678 & 0.02396 & 0.001 \\
Microbiome x Diet & 1 & 4.959 & 4.9593 & 91.863 & 0.07173 & 0.001 \\
Fly genotype x Microbiome x & & & & & & \\
Diet & 1 & 1.163 & 1.163 & 21.543 & 0.01682 & 0.001 \\
Tube (random effect) & 39 & 28.451 & 0.7295 & 13.513 & 0.41153 & 0.001 \\
Residuals & 213 & 11.499 & 0.054 & 0.16633 & &
\end{tabular}


Total

25969.135

1

Table 10: Analysis of deviance for Cox model with interactions between fly genotype, microbiome, and diet in the microbiome transplant.

\begin{tabular}{lrrrr}
\hline Category & \multicolumn{1}{l}{$\begin{array}{l}\text { Log- } \\
\text { likelihood }\end{array}$} & $X^{2}$ & Df & \multicolumn{2}{c}{$P$ value } \\
\hline Null & -22850 & & & \\
Fly genotype & -22716 & 268.8227 & 1 & $<2.20 \mathrm{E}-16$ \\
Microbiome & -22475 & 480.3245 & 2 & $<2.20 \mathrm{E}-16$ \\
Diet & -21846 & 1258.5088 & 1 & $<2.20 \mathrm{E}-16$ \\
Fly genotype x Microbiome & -21819 & 53.3452 & 2 & $2.61 \mathrm{E}-12$ \\
Fly genotype x Diet & -21817 & 4.2178 & 1 & 0.04 \\
Microbiome x Diet & -21807 & 20.0385 & 2 & $4.45 \mathrm{E}-05$ \\
Fly genotype x Microbiome x Diet & -21807 & 0.4244 & 2 & 0.8088
\end{tabular}

Table 11: Fixed effects from the mixed model of lifetime fecundity in the microbiome transplant. Significance was evaluated using Type III Wald F tests with Kenward-Roger degrees of freedom.

\begin{tabular}{|c|c|c|c|c|}
\hline Category & $\mathrm{F}$ & Df & Df.res & $P$ value \\
\hline Intercept & 46.6596 & 1 & 60.701 & 4.62E-09 \\
\hline Fecundity time point & 49.4043 & 2 & 105.269 & $7.38 \mathrm{E}-16$ \\
\hline Fly genotype & 0.0266 & 1 & 48.831 & 0.87103 \\
\hline Microbiome & 4.3529 & 2 & 49.038 & 0.01819 \\
\hline Diet & 5.4115 & 1 & 48.952 & 0.02419 \\
\hline
\end{tabular}




\section{SUPPLEMENTARY METHODS}

Microbiome transplant methods

To test if the microbiome can transfer adaptive traits, we performed a reciprocal microbiome transplant experiment (Fig. 1). All host $x$ microbiome $x$ diet interactions were generated in the reciprocal transplant. Both genotypes ( $\mathrm{C}$ and HS flies) were inoculated with microbiomes (sterile, $\mathrm{C}, \mathrm{HS}$ ) in both diets ( $\mathrm{C}$ and HS) for a total of 12 treatments. This allowed us to investigate how host $x$ microbiome $x$ environment interactions influence the transfer of adaptive phenotypes.

To transplant the microbiome, we collected fly frass from $300 \mathrm{C}$ and $300 \mathrm{HS}$ age-matched flies (7-10 days old). Donor flies were placed in sterile $50 \mathrm{ml}$ tubes lined with a plastic sheet and were allowed to defecate for $\sim 5 \mathrm{hrs}$. After $5 \mathrm{hrs}$, flies were immobilized at 4 으 $\mathrm{C}$ and the plastic sheet removed. Fly frass was washed off the plastic using sterile water to make a fecal solution. Because of large differences in the abundance of bacteria between $\mathrm{C}$ and HS flies (Supp. Fig. 7), C frass was resuspended in 6 ml, while HS frass was resuspended in $3 \mathrm{ml}$. The fecal solution was passed through a fine mesh to remove any fly eggs and other miscellaneous fly material. $50 \mu \mathrm{l}$ of fecal solution (or sterile water for the sterile treatments) was applied to axenic $\mathrm{C}$ and $\mathrm{HS}$ fly eggs onto sterile $\mathrm{C}$ and $\mathrm{HS}$ diets ( $7.5 \mathrm{ml}$ food in $50 \mathrm{ml}$ tube). Axenic eggs were prepared following standard procedures [1]. Sterile diets were prepared like the normal maintenance diets, but without acid and autoclaved. Flies were then reared in a biosafety cabinet at 25으 $\mathrm{C}$ and 12:12 light:dark cycle through the lifespan of the recipient flies. 5-6 replicates of each treatment were maintained and assessed for microbiome and phenotypic effects.

\section{Assessing fidelity of the microbiome transplant}

To determine the fidelity of the microbiome transplant, we performed 16S rRNA sequencing on the donor flies, frass, and adult recipient flies. All flies were age matched 7-10 days. Individual flies were sequenced. In brief, the 16S V1-V2 region was amplified, pooled, and digested with BstZ17I to deplete Wolbachia reads [2], and libraries were sequenced using 300 bp paired-end reads using the Illumina MiSeq platform at the Princeton University Genomics Core. We used QIIME2 v2019.4 to cluster into amplicon sequence variants (ASVs) with DADA2 and assign taxonomy with Greengenes classifier trimmed to the $16 \mathrm{~S}$ rRNA V1-V2 region. Data was imported into phyloseq for visualization and statistical analyses [3]. 
Flies with fewer than 500 reads were discarded from the analysis. Relative abundance of genera was compared across levels of the transplant (i.e., donor vs. frass vs. recipient). To assess how community structure varied in response to the microbiome treatment, beta-diversity measures were calculated with subsampling down to 500 reads/fly. Microbiomes were visualized with principal coordinate analysis (PCoA) using Bray-Curtis dissimilarity from ASVs. We tested if dispersion differed between the levels of transplant using a permutation test for homogeneity of multivariate dispersion. Because of significant differences in variance between the transplant levels, we focused on differences between groups within the recipients. We performed an ADONIS test to assess how interactions between fly genotype, microbiome, and diet influence the microbiome of recipients.

\section{Effects of the microbiome transplant on fly phenotypes}

First, we measured development time in the recipients. We counted the number of new pupae every 6 hours during their day time (i.e., $8 \mathrm{am}, 2 \mathrm{pm}, 8 \mathrm{pm}$ ) until all treatments began eclosing. We used the survival [4] and survminer [5] packages in $\mathrm{R}$ to analyse development data. We used Cox proportional hazards to identify how interactions between fly genotype, microbiome, and diet influence development time.

Second, we measured fecundity over the lifespan of recipients. Five females and five males from each replicate were transferred to new tubes. Flies were allowed to lay eggs for 24 hours and then flipped onto new food. Fecundity was measured at three timepoints over 34 days. Flies were flipped every 3-4 days in between fecundity measurements to avoid crowding and competition with developing larvae. Pupae were counted as a measure for fecundity and then divided by the number of females that survived to each fecundity measurement. We then analysed the effects of time, fly genotype, microbiome, and diet on fecundity with tube as a random effect using Ime4 [6]. We tested for significance of fixed effects using Type III ANOVA with Kenward-Roger degrees of freedom using the car package in R [7]. We tested if including interactions significantly improved the model fit by using a likelihood ratio test.

\section{SUPPLEMENTARY RESULTS}

\section{Microbiome transplant results}


To test if transferring the microbiome could transfer adaptive phenotypes to non-evolved hosts, we performed a fully reciprocal, host genotype $\mathrm{x}$ microbiome $\mathrm{x}$ diet transplant experiment. We assessed the fidelity of the microbiome transplant using $16 \mathrm{~S}$ rRNA sequencing of the donors, frass, and recipients. For both $\mathrm{C}$ and HS flies, the frass (which inoculated the diet of the recipients) was composed of fewer bacterial genera than found in donors (Supp. Fig. 1). The HS donor, frass, and recipient were dominated by Acetobacter species.

To compare how bacterial communities were structured across donor, frass, and recipient, we performed ordination analysis on Bray-Curtis distance matrix (Supp. Fig. 1B). The different steps of the transplant have different centroid dispersion (Supp. Table 6, $N_{\text {perm }}=999, F_{5,334}=36.862, p<0.001$ ). Broadly, the dispersion was greatest for recipients overall (Supp. Fig. 1C). For C flies, dispersion in frass microbial communities is greatly reduced compared to both donors and recipients. However, on HS flies, dispersion in frass and donors is similar, but lower than for recipients. The results from the dispersion analysis suggest that a subset of the microbiome was transferred through the frass, and for the recipients, differences in the structure of microbiome communities accumulated through their lifespan.

Both fly genotype and diet shaped the fidelity of the microbiome transplant in the recipients. Using Bray-Curtis distance to examine microbiome differences in recipients, we find that both microbiome and diet strongly impact beta-diversity in the recipients (Supp. Fig. 1D). On the $C$ diet, recipients cluster by microbiome received (Supp. Table 7, $F_{1,143}=111.45, r^{2}=0.43, p=0.001$ ), suggesting a successful microbiome transplant. However, on the HS diet, the microbiome transplant breaks down and only had weak, though significant effects (Supp. Table 8, $\mathrm{F}_{1,115}=4.35, \mathrm{r}^{2}=0.04, \mathrm{p}=0.003$ ). Overall, diet explained $14 \%$ and microbiome explained $15 \%$ of variation in microbial communities, while interactions between fly, microbiome, and diet had significant, but weak effects $\left(r^{2}<8 \%\right.$, Supp. Table 9). For example, $\mathrm{C}$ flies that receive the $\mathrm{C}$ microbiome contained Acetobacter, Commensalibacter, and Gluconobacter, whereas HS flies only contained Acetobacter (Supp. Fig. 1D). Thus, the HS diet potentially selected for associations between fly and Acetobacter. Overall, despite the deviation from the donor microbiomes (Supp. Fig. 1C), the microbiome transplant led to different microbiomes between $\mathrm{C}$ and $\mathrm{HS}$ recipients, especially on the $\mathrm{C}$ diet. 
Microbiome transplant shapes life history traits, but depends on the environment

The microbiome significantly shaped developmental time, but depended on fly genotype and diet (Supp. Fig. $2 \mathrm{~A}$, concordance $=0.825$, log-rank test $\mathrm{p}<0.0001$ ). On the $\mathrm{C}$ diet, both fly genotypes responded to the microbiome in a similar way: sterility slowed development (avg. $136.9+/-0.41 \mathrm{hrs}$ ), while C and HS microbiomes accelerated development similarly (C avg. 119.8 +/- 0.49 hrs, HS avg. 121.2 +/- 0.49 hrs). However, on the HS diet, the microbiome shaped development in different ways. The C microbiome (avg. 135.7 +/- $0.59 \mathrm{hrs}$ ) accelerated development compared to the HS microbiome (avg. 142.26 +/- $0.52 \mathrm{hrs}$ ) and the sterile treatments were the slowest (avg. $167.8+/-0.73 \mathrm{hrs}$ ). Two-way interactions between fly genotype, microbiome, and diet significantly shaped development time, but the three-way interaction did not (Supp. Table 10). The model with interactions best explained development data compared to one without interactions ( $\log$-likelihood $\left.=-21807, X^{2}=78.02, d f=7, p<0.0001\right)$. Overall, the results from development suggest that the microbiome and diet contribute to fly development.

To approximate fitness consequences of the microbiome transplant, we measured fecundity over the lifespan of the recipients (Supp. Fig. 2B). Fecundity declined over time $\left(F_{2,101.4}=46.6, p<0.0001\right)$. The decline in fecundity was modified by both the diet and microbiome, but not by fly genotype (Supp. Table 11). Diet reduced fecundity by $26 \%$ in the HS diet (C diet avg. 26.3 +/- 2.40, HS diet avg. 19.8 +/- 1.69 pupae/female). The microbiome also affected fecundity, but only with the sterile flies having significantly lower fecundity (sterile avg. 15.4 +/-2.25, C avg. 24.5 +/- 2.42, HS avg. 26.1 +/- 2.56). The interaction between microbiome and time was significant $\left(\mathrm{F}_{4,101.3}=5.84, \mathrm{p}=0.0003\right)$, driven by the slower decline in fecundity for the sterile treatments. The model that included this interaction best explained variation in fecundity (log-likelihood = $644.3, X^{2}=22.44, p=0.0002$ ). The results from the fecundity trials suggest that the presence of a microbiome can modify fly fitness on the HS diet, but little difference was observed between the C and HS microbiomes.

\section{SUPPLEMENTARY REFERENCES}

1. Koyle ML, Veloz M, Judd AM, Wong ACN, Newell PD, Douglas AE, et al. Rearing the Fruit Fly Drosophila melanogaster Under Axenic and Gnotobiotic Conditions. 2016; e54219.

2. Simhadri RK, Fast EM, Guo R, Schultz MJ, Vaisman N, Ortiz L, et al. The Gut Commensal Microbiome of Drosophila melanogaster Is Modified by the Endosymbiont Wolbachia. mSphere. 2017;2. 
doi:10.1128/msphere.00287-17

3. McMurdie PJ, Holmes S. phyloseq: an R package for reproducible interactive analysis and graphics of microbiome census data. PLoS One. 2013;8: e61217.

4. Therneau TM, Lumley T. Package “survival.” R Top Doc. 2015;128: 112.

5. Kassambara A. Kosinski M. survminer: Drawing survival curves using "ggplot2," 2018. URL https://CRAN Rproject org/package= survminer R package version 0 4. 2018;3.

6. Bates D, Sarkar D, Bates MD, Matrix L. The Ime4 package. R package version. 2007;2: 74.

7. Fox J, Weisberg S, Adler D, Bates D, Baud-Bovy G, Ellison S, et al. Package "car." Vienna: R Foundation for Statistical Computing. 2012. Available: https://cran.microsoft.com/snapshot/2017-06-

17/web/packages/car/car.pdf 